\title{
Distance Protection for Coexistence of 5G Base Station and Satellite Earth Station
}

\author{
Yiqiao Wei ${ }^{1}$, Shuzhi Liu ${ }^{2}$ and Seung-Hoon Hwang ${ }^{2, *(\mathbb{D})}$ \\ 1 China Mobile (Shanghai) Industrial Research Institute, Shanghai 200120, China; \\ weiyiqiao@cmsr.chinamobile.com \\ 2 Division of Electronics and Electrical Engineering, Dongguk University, Seoul 04620, Korea; \\ shuzhiliu@dongguk.edu \\ * Correspondence: shwang@dongguk.edu; Tel.: +82-2-2260-3994
}

Citation: Wei, Y.; Liu, S.; Hwang,

S.-H. Distance Protection for Coexistence of 5G Base Station and Satellite Earth Station. Electronics 2021, 10, 1481. https://doi.org/ 10.3390/electronics10121481

Academic Editor: Christos J. Bouras

Received: 26 May 2021

Accepted: 17 June 2021

Published: 19 June 2021

Publisher's Note: MDPI stays neutral with regard to jurisdictional claims in published maps and institutional affiliations.

Copyright: (c) 2021 by the authors. Licensee MDPI, Basel, Switzerland. This article is an open access article distributed under the terms and conditions of the Creative Commons Attribution (CC BY) license (https:// creativecommons.org/licenses/by/ $4.0 /)$.

\begin{abstract}
In this paper, we investigate the coexistence of the 5G communication network with a fixedsatellite service (FSS) in the $3.5 \mathrm{GHz}$ and $26 \mathrm{GHz}$ frequency bands. We analyze a distance protection scheme for the FSS Earth station (ES) and 5G base stations (BS). Furthermore, we define the exclusion and restriction zones to develop a transmit power control scheme based on the Citizens Broadband Radio Service (CBRS). An interactive power control scheme is also devised for the restriction zone and extensively analyzed through simulations. The proposed scheme is examined for practical scenarios such as the rural macrocells (RMa), urban macrocells (UMa), and urban microcells (UMi) as defined by the 3GPP. The impact of the antenna type is also investigated, and BSs with omnidirectional, $4 \times 4$, $8 \times 8$, and $16 \times 16$ antenna arrays are examined, as defined by $3 \mathrm{GPP}$, for the $5 \mathrm{G}$ networks. The results confirm that $5 \mathrm{G}$ systems can coexist with the FSS and provide quantitative insights into the selection of the system parameters, including interference margins, exclusion sizes, and reduction zones, for different scenarios and antenna types.
\end{abstract}

Keywords: 5G system; fixed satellite systems; coexistence/sharing scheme; distance protection

\section{Introduction}

The demand for fifth generation (5G) radio frequency $(\mathrm{RF})$ communications continues to grow at an alarming rate; as a new generation of mobile communication technology, $5 \mathrm{G}$ has higher data rates, shorter delays, and larger numbers of connections than 4G. The 5G technology utilizes 'high', 'medium', and 'low' frequencies [1]; therefore, it can exploit the specific characteristics of different frequency bands of the spectrum based on usage scenarios. For instance, low frequencies (below $2 \mathrm{GHz}$ ) will continue to be essential for extending the $5 \mathrm{G}$ mobile broadband experience to wide areas and deep indoor environments. On the other hand, high frequencies (above $6 \mathrm{GHz}$ ) will prove indispensable for providing additional capacity and delivering extremely high data rates required by some of the $5 \mathrm{G}$ enhanced mobile broadband (eMBB) applications. Furthermore, medium bands $(2-6 \mathrm{GHz})$ are suitable for managing the trade-offs between extended coverage and high capacity.

In 2018, the Republic of Korea completed auctions of the $5 \mathrm{G}$ frequency bands, namely the $3.42-3.70 \mathrm{GHz}$ and $26.5-28.9 \mathrm{GHz}$ bands, as shown in Figure 1 [1]. In the future, additional frequencies may be allocated from the adjacent frequency bands; however, there are incumbent users in the existing bands, such as fixed-satellite service (FSS) [2,3], intersatellite services, Earth-exploration-satellite service, and meteorological-satellite service [4]. UK Ofcom has identified the 3.8-4.2 GHz band to have the potential for increased sharing and is considering the development of proposals to facilitate shared-frequency access in this band. In August 2018, the FCC of the USA adopted a Notice of Proposed Rulemaking to release the 3.7-4.2 GHz band for new wireless services, while accommodating incumbents, and to seek comments on proposals to promote more spectrum-efficient, and intensive, 
fixed use of the band on a shared basis. Previously, several studies have been conducted on GSM to access the current utilization of the C-band (3.5-4.2 GHz) to define a strategy and roadmap to release this band to support the deployment of $5 \mathrm{G}$ services. This research has been, and is being, conducted to ensure independent coexistence with other C-band users [5].

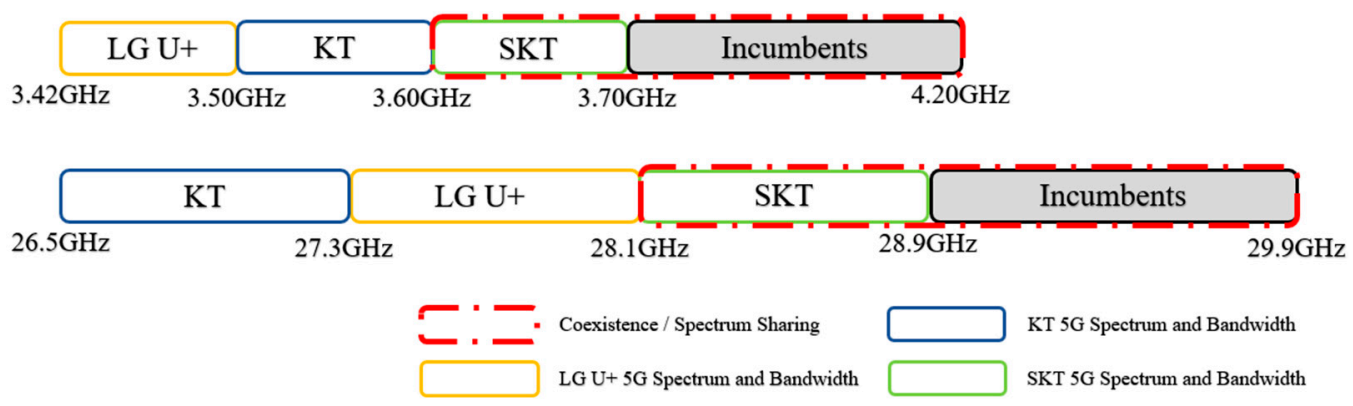

Figure 1. Korea's 5G frequency allocations and their adjacent bands [1].

The interference between two communication systems is the main problem of the coexistence of two systems in one frequency band. There are four main types of interference between $5 \mathrm{G}$ and FSS systems: interference from $5 \mathrm{G}$ base stations (BSs) to FSS Earth stations, 5G users to FSS Earth stations (ESs), FSS devices to 5G BSs, and FSS devices to 5G users. Figure 2 shows the coexistence relationship between the FSS and cellular systems. The signal transmission paths from the space station (SS) to the ESs constitute the downlinks of the FSS system, mainly in the 3.7-4.2 GHz band, and the signal transmission paths from the ESs to a SS constitute the uplinks of the FSS system mainly in the 29.1-29.9 GHz band. In Figure 2, (1) is the signal transmission path from a BS to an ES, and the ES interferes with the $5 \mathrm{G}$ system that must be protected [2,6,7]; (2) is the signal transmission path from the user equipment (UE) to the ES, and the downlink from the SS to the ES should also be protected $[2,6]$. For the interference experienced by the 5G BS/UE from the SS, (3) and (4) are downlinks from the SS to the BS and UE, but it has not been varified whether (3) and (4) can coexist. The uplink from the ES to SS operates in the $29.1-29.9 \mathrm{GHz}$ band, and it can coexist with the links (5) and (6) [8]; however, (7) and (8), which are the interference from the ES to BS and UE, should be protected [8,9]. In this study, we focus on minimizing (1), the inteference from the BS to the ES. The main problem should be solved, to achieve the coexistence between the FSS and 5G cellular, under the following considerations: Furthermore, compared with (2), the 5G gNBs utilizes massive multi-input multi-output (MIMO) systems, and the FSS ESs use antenna dishes with high directivities and antenna gains. This could lead to the alignment/misalignment of their respective antenna directions [2]. Besides, the interference signals generated and observed at the BSs are more significant than those at the UEs [3] because the equivalent isotopically radiated power of the UE is likely to be much lower than that of a BS, and the UE has a lower probability of line-of-sight (LOS) propagation towards the ES/SS [8]. In a system with the coexistence of FSS and cellular systems, we can restrain the transmission characteristics of the BSs to protect the FSS downlinks, e.g., by controlling the transmit power and/or beamforming. However, for $(7)$, it is infeasible to restrain the ES. 


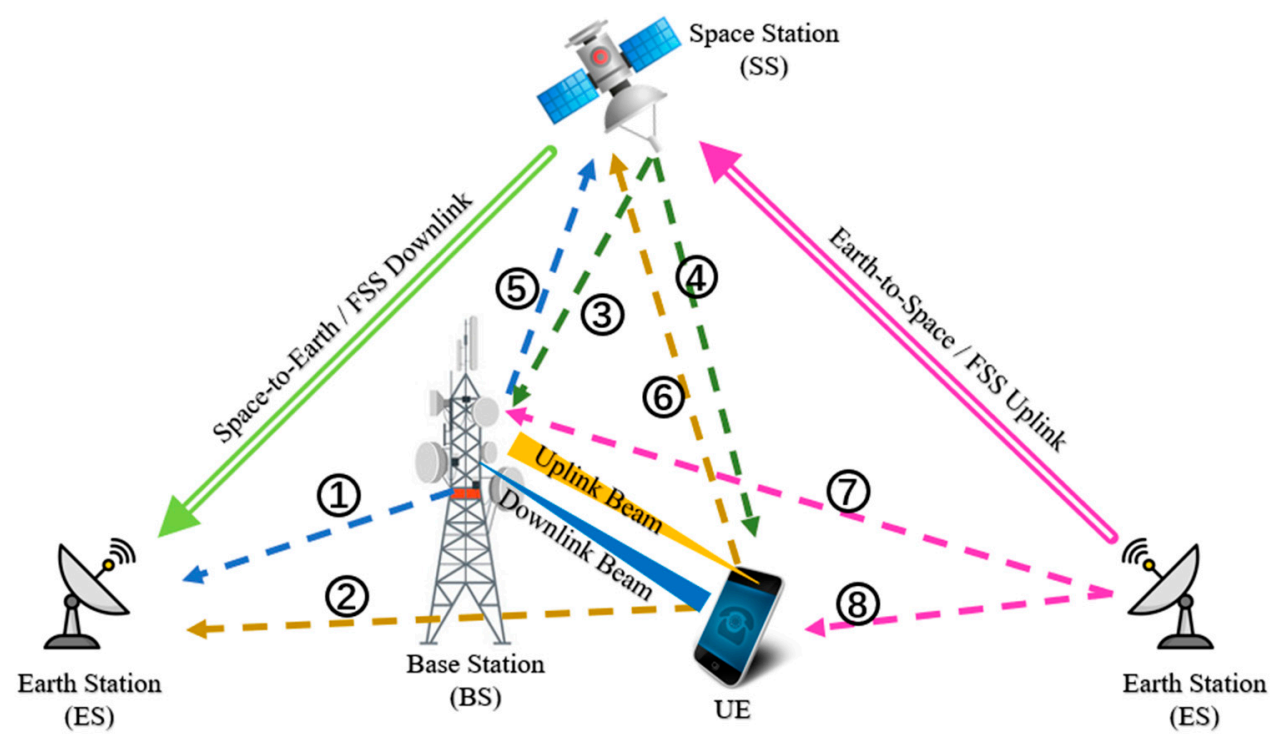

Figure 2. Coexistence of the FSS and cellular systems.

The existing work was mainly focused on the release of new frequency bands to support 5G rollout. Two methods were proposed in [10] to enable administrations to protect existing users in the 3.6-3.8 GHz band while allowing mobile/fixed communication networks to use them as new entrants. In addition, reference of [11] mainly analyzed different BS/ES deployment schemes, antenna configurations, and transmitter parameters, so as to utilize the characteristics of the millimeter wave frequency band and study the coexistence between cellular and satellite services. In the current coexistence schemes, passive interference mitigation techniques are often utilized as they do not require coordination with the incumbent systems. Space domain method is the most popular method and is adopted in most coexistence studies. It can be further divided into exclusion zones (i.e., distance isolation protection) and restriction zones. This scheme is based on the interference/noise $(\mathrm{I} / \mathrm{N})$ protection threshold technique, which involves a maximum permitted interference at the input to the FSS ES. A protection distance is set between the $5 \mathrm{G}$ systems and FSS to form an exclusion zone $[2,7,8,10,12]$ where none of the BSs can be deployed in these exclusion zones. Additionally, restriction zones and BS transmit (Tx) power control schemes, in the restriction zone, are studied under the distance protection method. The advantages of this scheme are that the layout is simple, coexistence efficiency is high, and the scheme can be adapted to most environments.

In this study, we investigate the limitations of the FSS protection methods. In most of the aforementioned methods, only a single-region deployment scenario is considered. Furthermore, in most of the proposed methods, the effects of the ES antenna elevation angle on the different protection methods have been overlooked. The techniques to determine the zones and power control schemes, with the restriction zones, are also not investigated in detail. In this work, we address these issues and propose a method to define both the exclusion and restriction zones for space domain protection. Inspired by the power control schemes used in the Citizens Broadband Radio Service (CBRS), we propose and study some interactive power control schemes in the restricted areas. The main contributions of this work are as follows:

- Using various parameters, provided by the 3GPP and ITU, we show that our proposed method covers all rural macrocell (RMa), urban macrocell (UMa), and urban microcell (UMi) deployment scenarios defined by 3GPP. We further investigate the impact of the antenna pattern defined by the 3GPP omnidirectional antenna and various 5G large-antenna arrays (i.e., $4 \times 4,8 \times 8$, and $16 \times 16$ ).

- We investigate the impact of the ES antenna elevation angle on different protection methods and consider the worst-case scenario for FSS ES protection. We further 
incorporate the LOS and non-line-of-sight (NLOS) propagation conditions between the BS and ES using the 3GPP LOS probability function.

- We propose a new protection method, which combines the exclusion area and restricted area, where the BSs subjected to Tx power control scheme could be deployed. Moreover, we propose an iterative BS Tx power control scheme. The proposed protection method, using the exclusion area as well as restricted areas, with Tx power control scheme are simulated.

The main differences in methods, proposed schemes and considerations between our work and previous works are summarized in Table 1.

Table 1. The main differences between our work and previous works.

\begin{tabular}{|c|c|c|c|c|c|c|c|c|c|}
\hline & & Our Work & [2] & [3] & [6] & [7] & [8] & [9] & [12] \\
\hline \multirow{4}{*}{$\begin{array}{l}\text { Protection schemes } \\
\text { studied }\end{array}$} & Exclusion Zone & o & $\mathrm{o}$ & & & & $\mathrm{o}$ & & o \\
\hline & Restriction Zone & o & o & o & o & & $\mathrm{o}$ & & o \\
\hline & $\begin{array}{l}\text { Restriction Zone with Pover } \\
\text { Control Scheme }\end{array}$ & o & & & & & & & \\
\hline & $\begin{array}{l}\text { Combination of Exclusion } \\
\text { Zone \& Exclusion Zone }\end{array}$ & o & & & & & & & \\
\hline \multirow{3}{*}{$\begin{array}{l}\text { Deployment } \\
\text { scenarios considered }\end{array}$} & Rma & o & & & & o & $\mathrm{o}$ & & \\
\hline & Uma & o & & & & o & $\mathrm{o}$ & & \\
\hline & Umi & o & o & & & & o & & \\
\hline \multicolumn{2}{|c|}{ The effect of different ES Antenna elevation angle } & o & & & & & & & \\
\hline \multirow{4}{*}{$\begin{array}{l}\text { Antenna types } \\
\text { studied }\end{array}$} & Omni & o & & o & & & o & o & o \\
\hline & $4 \times 4$ & o & & o & & & $\mathrm{o}$ & & \\
\hline & $8 \times 8$ & $\mathrm{o}$ & & o & & & o & $\mathrm{o}$ & \\
\hline & $16 \times 16$ & $\mathrm{o}$ & & & & & o & o & \\
\hline
\end{tabular}

The remainder of this paper is organized as follows. The basic scenarios and simulation parameters are described in Section 2. The interference derivations are presented in Section 3. The simulated and experimental results for the proposed system are presented, and discussed, in Section 4. Finally, we present our conclusions and an outline of our future research in Section 5.

\section{Background Knowledge}

\subsection{Basic Scenarios and Parameters}

Both the azimuth and elevation planes need to be considered because the antenna gain is closely related to both azimuth and elevation off-boresight angles. Hence, they have a significant impact on the interference from the BS to ES. For the protection target, i.e., the FSS ES, the ES is assumed to be located at the center of the area with a coordinate of $(0,0)$. Further, the azimuthal orientation of the ES antenna is assumed to be true north, while the ES antenna elevation orientation, i.e., elevation angle, differs for various ESs, according to the ES deployment requirements. The cellular BS (eNodeB/gNodeB) is assumed to be deployed at any possible location $(X, Y)$ around the ES. Moreover, the azimuthal orientation of the BS antenna is assumed to be true south. Herein, we use the 3-dimensional $(X, Y, Z)$ coordinate to present the results. In this coordinate system, the $\mathrm{x}$ and $\mathrm{y}$ axes are the latitude and longitude coordinates of the BSs, respectively, and the z-axis is the target measurement, such as ES antenna gain, path loss, and I/N, with the line between the BS and ES as the interference axis.

We consider three scenarios, including RMa (rural), UMa (urban), and UMi (urban) [13]. The ES antenna heights and their diameters as well as the BS antenna heights 
are different for these three scenarios. Further, the inter-site distances (ISDs), propagation losses, shadow fading, and LOS/NLOS probability distributions are different for the three scenarios. The carrier frequency is set to the central frequency of the $3.7-4.3 \mathrm{GHz}$ band, i.e., $3.95 \mathrm{GHz}$, as shown in Figure 3. For setting the basic scenarios, we use various simulation parameters. The FSS ES simulation parameters for all the considered scenarios are presented in Tables 2 and 3.

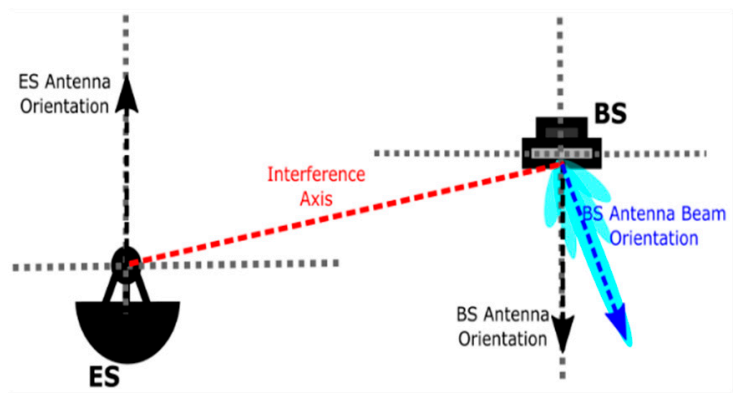

(a) azimuth plane

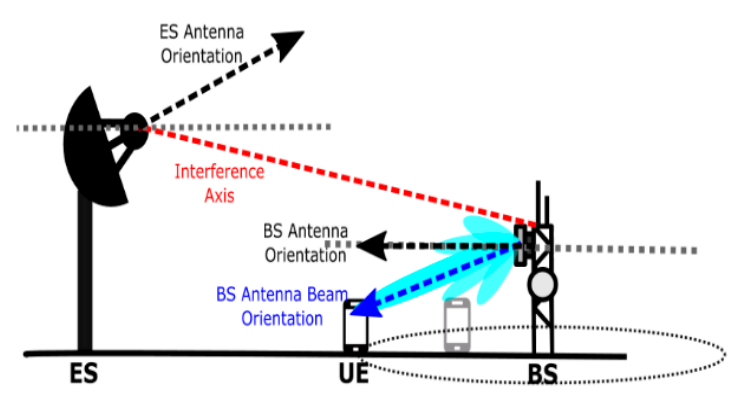

(b) elevation plane

Figure 3. Carrier frequency set to the center frequency in the (a) azimuthal and (b) elevation planes.

Table 2. FSS Earth Station (ES) parameters [13-15].

\begin{tabular}{ccccccc}
\hline & RMa & UMa & UMi & RMa & UMa & UMi \\
\hline ES antenna diameter $(\mathrm{m})$ & 10 & 2.4 & 2.4 & Thermal noise $(\mathrm{dBm} / \mathrm{Hz})$ & -179 \\
\hline ES antenna pattern & Recommendation ITU-R S.465-6 & ES bandwidth $(\mathrm{MHz})$ & 36 \\
\hline ES antenna height $(\mathrm{m})$ & 7 & 30 & 30 & $\begin{array}{c}\text { ES antenna maximum gain } \\
(\mathrm{dBm})\end{array}$ & 56 \\
\hline $\begin{array}{c}\text { ES antenna elevation } \\
\text { angle (degrees) }\end{array}$ & $5^{\circ}, 15^{\circ}, 48^{\circ} / 27.5^{\circ}$ mean value & $\begin{array}{c}\text { ES antenna adjacent channel } \\
\text { sensitivity (ACS) model }\end{array}$ & Recommendation ITU-R S.465-6 \\
\hline $\begin{array}{c}\text { ES receiving system } \\
\text { noise temperature }(\mathrm{K})\end{array}$ & 100 & Effective Earth radius (m) & $8.5 \times 10^{6}$ \\
\hline
\end{tabular}

Table 3. Cellular BS and environmental parameters [14-16].

\begin{tabular}{|c|c|c|c|c|c|c|c|c|}
\hline & & RMa & UMa & UMi & & RMa & UMa & UMi \\
\hline \multicolumn{2}{|c|}{ BS antenna height (m) } & 35 & 25 & 10 & BS channel bandwidth (MHz) & & 20 & \\
\hline \multicolumn{2}{|c|}{ UE height (m) } & 1.5 & 1.5 & 1.5 & Effective environment height (m) & 1 & None & None \\
\hline \multicolumn{2}{|c|}{ Inter-site distance, ISD (m) } & 1732 & 500 & 200 & Average building height (m) & 5 & None & None \\
\hline \multicolumn{2}{|c|}{ BS maximum Tx power $(\mathrm{dBm})$} & 46 & 38 & 38 & Average street width (m) & 20 & None & None \\
\hline \multirow{3}{*}{$\begin{array}{l}\text { BS antenna Tx } \\
\text { gain }(\mathrm{dB})\end{array}$} & Omni antenna & 15 & 15 & 15 & LOS shadow fading $(\mathrm{dB})$ & 6 & 4 & 4 \\
\hline & \multirow{2}{*}{$\begin{array}{l}\text { Single antenna element } \\
\text { in MIMO antenna array }\end{array}$} & \multirow{2}{*}{8} & \multirow{2}{*}{8} & \multirow{2}{*}{8} & NLOS shadow fading $(\mathrm{dB})$ & 8 & 6 & 7.82 \\
\hline & & & & & BS minimum coupling loss $(\mathrm{dB})$ & 80 & 70 & 70 \\
\hline
\end{tabular}

\subsection{Interference Derivation}

A directional antenna produces a tapered main beam containing most of the radiated power. In this work, we mainly focus on the interference calculations in the azimuthal and elevation planes. The interferences in these planes are given by

$$
I_{F S S E S \rightarrow B S}=P_{B S t x}+G_{F S S E S}+G_{B S}-P L
$$


where $P_{B S t x}$ is the transmit station signal power density in $\mathrm{dBm} / \mathrm{MHz}[17,18], G_{F S S}$ ES represents the antenna gain of the ES in the direction of the transmit station in $\mathrm{dBi}$ [11], and $G_{B S}$ is the $5 \mathrm{G}$ BS antenna gain, with an omnidirectional antenna and massive MIMO antenna array in the direction of the ES to account for the beamforming antenna pattern in $\mathrm{dBi}[14,19]$, and PL is the combined path loss from all possible BS locations to the ES [14]. The FSS ES antenna gain is also described.

Spectrum management of the frequency bands shared by the FSS ESs and fixed terrestrial radios may require the calculation of the terrestrial emitter RF power at the input of an ES receiver [20]. This is to ensure that the ES interference threshold is not exceeded. The potential received interference power from terrestrial emitters is partly dependent on the FSS ES antenna gain in the direction of the terrestrial emitter. The FSS ES antenna gain is calculated as follows:

- $\quad$ ES antenna pattern: Recommendation ITU-R S.465-6 [11]

$$
\begin{gathered}
G_{E S}(\varnothing)=\left\{\begin{array}{c}
G_{\max }, \text { if } 0^{\circ}<\varnothing<\varphi_{\min } \\
32-25 \log \varnothing, \text { if } \varphi_{\min } \leq \varnothing<48^{\circ} \\
-10, \text { if } 48^{\circ} \leq \varnothing \leq 180^{\circ}
\end{array}\right. \\
\varphi_{\text {min }}=\left\{\begin{array}{c}
\operatorname{Max}\left(1^{\circ}, 100 \lambda / D\right), \text { if } D / \lambda \geq 50 \\
\operatorname{Max}\left(2^{\circ}, 114(\lambda / D)^{-1.09}\right), \text { if } D / \lambda<50
\end{array}\right.
\end{gathered}
$$

The off-axis / off-boresight angle, $\varnothing$ i.e., the angle between the interference axis and direction of the main lobe (ES antenna orientation) of the FSS ES (ITU-R P.452-15 [15]) is given as

$$
\begin{gathered}
\varnothing=\arccos (\cos (\alpha) \cos (\epsilon) \cos (\vartheta)+\sin (\alpha) \sin (\epsilon)) \\
\epsilon=\frac{h_{E S}-h_{B S}}{d}-\frac{d}{2 r}
\end{gathered}
$$

where $\vartheta$ : azimuth angle of the BS w.r.t the ES main lobe, $\alpha$ : ES elevation angle, $r$ : effective Earth radius $=8.5 \times 10^{6} \mathrm{~m}$, and $\lambda$ : wavelength. Since the ES antenna orientation is set to true north, areas closer to the main lobe of the ES antenna receive higher antenna gain. The ES is considered to be at the center $(0,0)$, and the $\mathrm{X}-, \mathrm{Y}$-axis values are the BS coordinates. Figure 4 shows the ES antenna gain versus the BSs locations.

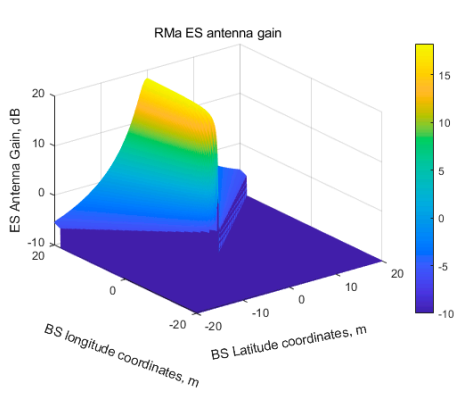

(a) RMa ES Antenna Gain

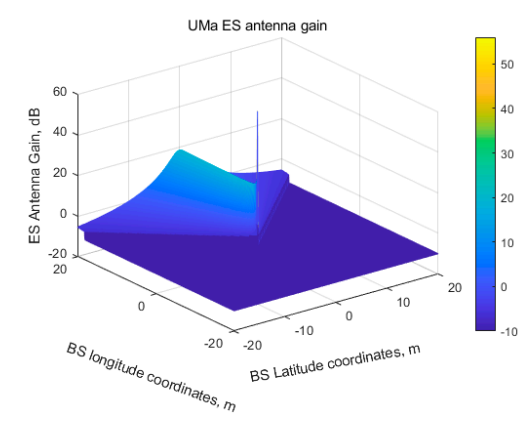

(b) UMa ES Antenna Gain

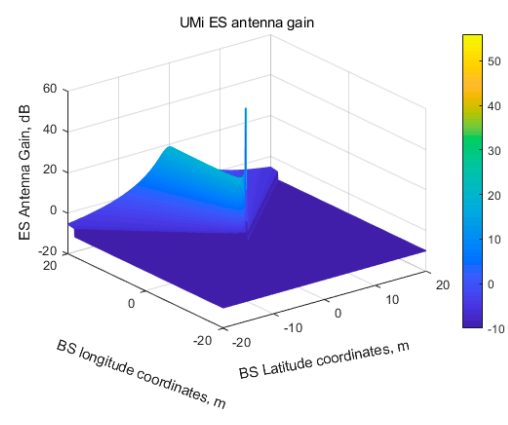

(c) UMi ES Antenna Gain

Figure 4. FSS ES antenna gain orientations.

The path losses from all possible BS locations to the ES are next considered. To calculate the path loss, we adopt the 3D propagation model proposed by the 3GPP in [14], which is often referred to as the 3GPP new radio (NR) model. This model is compliant with earlier channel models, e.g., 3D SCM model [21] and IMT-advanced [22] up to $6 \mathrm{GHz}$. It also supports comparisons across the frequency bands over the range of $0.5-100 \mathrm{GHz}$ and various scenarios, including RMa, UMi, and UMa. The model in [14] considers 3D propagation distance; therefore, both the BS and ES antenna heights significantly affect 
the actual path loss values. In cellular network simulations, the path losses in rural areas are always much lower than those in urban areas; this is, mainly, because the altitudes of the Tx and receive $(\mathrm{Rx})$ antennas are the same for all scenarios. However, the ES and BS antenna heights are both different in the RMa, UMa, and UMi. The altitude differences between the ES and BS antennas are the largest in these three scenarios. The path loss for $\mathrm{RMa}$ is slightly higher than those of the UMa and UMi scenarios. Figure 5 shows the signal propagation losses from all possible BS locations $(X, Y)$ at the ES $(0,0)$. In practice, the LOS and NLOS scenarios are combined in the real environment, and the NLOS path is often the dominant loss component in urban areas. Therefore, we combine the LOS and NLOS path losses using a random mechanism and the LOS probability as shown in Algorithm 1, which is a function of the distance, as specified in the 3GPP model.

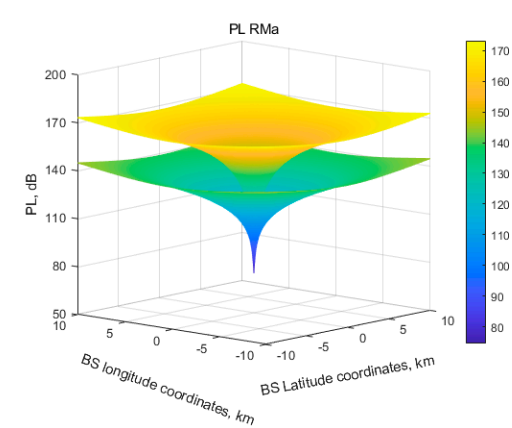

(a) Path Loss of RMa

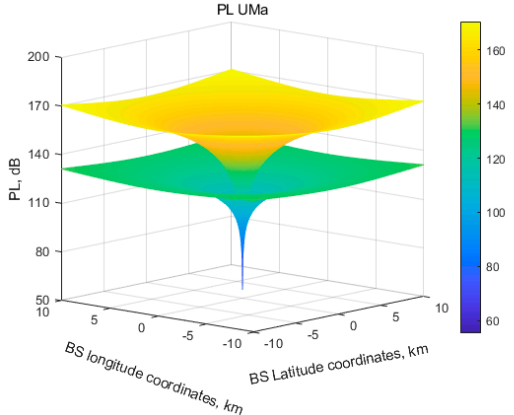

(b) Path Loss of UMa

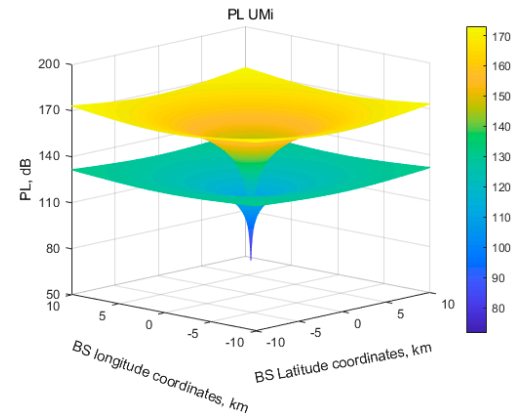

(c) Path Loss of Umi

Figure 5. Path loss propagation model.

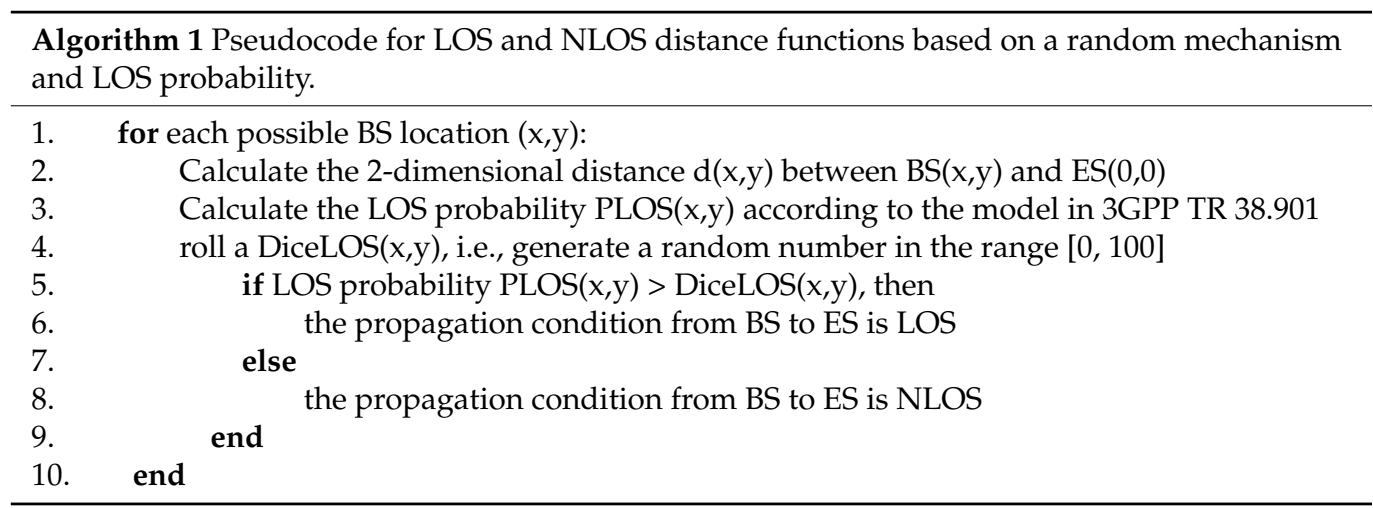

In obtaining the BS antenna gain, the g-NodeB antenna follows the 3GPP NR model [14], where the antenna elements are uniformly distributed in $\mathrm{N}$ columns with horizontal spacing of $\Delta H$ and in $\mathrm{M}$ rows with vertical spacing of $\Delta V$.

For a single antenna element radiation pattern, the BS antenna gain is calculated as:

- Horizontal pattern:

$$
A_{E, H}(\varphi)=-\min \left[12\left(\frac{\varphi}{\varphi_{3 d B}}\right)^{2}, A_{m}\right]
$$

- Vertical pattern:

$$
A_{E, V}(\theta)=-\min \left[12\left(\frac{\theta-90}{\theta_{3 d B}}\right)^{2}, S L A_{V}\right]
$$


- Single antenna element pattern:

$$
A_{E}(\varphi, \theta)=G_{E, M a x}-\min \left\{-\left[A_{E, H}(\varphi)+A_{E, V}(\theta)\right], A_{m}\right\}
$$

where $\varphi_{3 d B}=65^{\circ}$ : horizontal 3-dB beamwidth, $A_{m}=30 \mathrm{~dB}$ : front-to-back ratio, $\theta_{3 d B}=65^{\circ}$ : vertical 3-dB beamwidth, and $S L A_{V}=30 \mathrm{~dB}$ : side-lobe level limit. However, a planar uniform rectangular array (URA) antenna pattern uses the procedure illustrated below:

- Array factor:

$$
A F\left(\varphi, \theta, \varphi_{\text {beam }}, \theta_{\text {beam }}\right)=10 * \log _{10}\left[1+\rho\left(\left|a \cdot w^{T}\right|^{2}\right)\right]
$$

where $w=\left[w_{1,1}, w_{1,2}, \ldots, w_{m, m}\right], \mathrm{m}=\sqrt{N_{e}}, w_{p, r}=e^{j 2 \pi}\left((p-1) \frac{\Delta V}{\lambda} \Psi_{P}+(r-1) \frac{\Delta H}{\lambda} \Psi_{r}\right)$, $\left\{\begin{array}{c}\Psi_{P}=\cos (\theta)-\cos \left(\theta_{\text {beam }}\right) \\ \Psi_{P}=\sin (\theta) \sin (\varphi)-\sin \left(\theta_{\text {beam }}\right) \sin \left(\varphi_{\text {beam }}\right)\end{array}\right.$.

- Array pattern:

$$
A_{A}\left(\varphi, \theta, \varphi_{\text {beam }}, \theta_{\text {beam }}\right)=A_{E}(\varphi, \theta)+A F\left(\varphi, \theta, \varphi_{\text {beam }}, \theta_{\text {beam }}\right)
$$

where $\left(\varphi_{\text {beam }}, \theta_{\text {beam }}\right)$ : main beam steering angle due to beamforming, $\rho$ : correlation coefficient assumed to be equal to unity, $a$ : amplitude vector with an equal and fixed amplitude, $N e$ : number of antenna elements in the array, and $\Delta V=\Delta H=\lambda / 2$ : vertical and horizontal spacings between the array elements.

Using electronic steering, a planar URA can generate the main and side lobes. By increasing the number of elements in the antenna array, the main lobe beam can provide higher gain while creating a larger number of side lobes. The larger the number of elements in the antenna, the narrower is the main-lobe beam. However, the main-lobe beam may point in any direction, but the gain of the main-lobe beam decreases as the beam gradually deviates from the antenna array orientation. Figure 6 shows the antenna array gains of various $16 \times 16$ antenna arrays with different beam sweep angles.
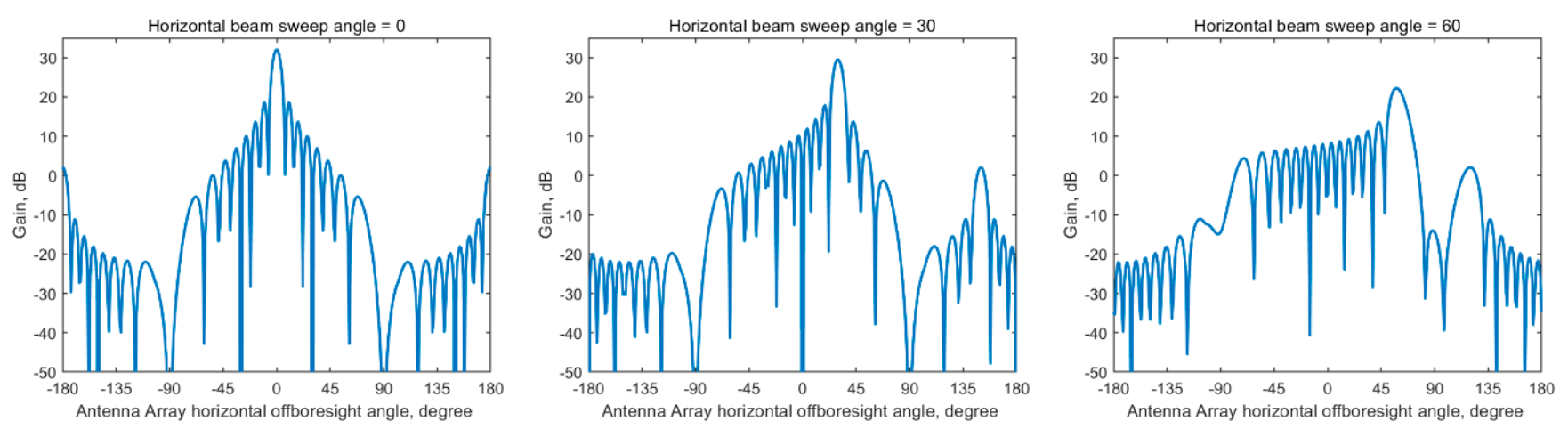

Figure 6. Antenna gains of a $16 \times 16$ antenna array.

According to [17,18], a Tx power limit of $38 \mathrm{dBm}$ is chosen for the medium-range BS, and no upper limit is considered for the wide-area BSs. The medium-range BS Tx power limit (38 dBm) is also chosen for the UMa and UMi, while a limit of $46 \mathrm{dBm}$ is chosen for the RMa scenario in the wide-area BS, according to the maximum macrocell BS Tx power defined in [16].

\section{Distance Protection Methods}

In this section, we present investigations of the functions of the distance protection method, including the I/N protection threshold, exclusion zone, restriction zone, and BS Tx power control scheme in the restriction zone. 


\subsection{I/N Protection Threshold}

In the distance protection method, there is a maximum permitted interference at the input to the FSS ES. The long-term interference criterion, defined in [13], is considered herein to access the interference from the cellular systems at the FSS ES receivers. For the long-term interference, however, two cases can be considered depending on the types of scenarios noted in recommendation ITU-R S.1432:

- In-band sharing:

(1) $\quad \mathrm{I} / \mathrm{N}=-12.2 \mathrm{~dB}$, corresponding to the total interference received from other systems having co-primary statuses during $100 \%$ of the worst month.

(2) $I / \mathrm{N}=-10 \mathrm{~dB}$, corresponding to the aggregate interference received from the co-primary allocations during $20 \%$ of any month.

- Adjacent band sharing:

(1) $\quad I / N=-20 \mathrm{~dB}$, corresponding to the aggregate interference received from all other sources during $100 \%$ of the time.

Based on the above criteria, in our simulation, we set $\mathrm{I} / \mathrm{N}=-12.2 \mathrm{~dB}$ as the in-band interference threshold.

\subsection{Exclusion Zone}

To obtain the exclusion zone in the distance protection method, the procedure shown in Figure 7 is followed. In calculating the interference from all base station locations $(x, y)$, we need to consider: the transmission power of the base station, the antenna gain of the base station, the antenna gain of the FSS ES, and the path loss. One also needs to make sure the interference $(x, y)$ is less than the I/N protection threshold to be able to use this model. If the interference $(x, y)$ meets the protection threshold, the base station can be deployed at this location. The location $(x, y)$ is then marked in the red color. Finally, all red location points from the exclusion zone are outlined. Secondly, in determining the value of the path loss, six different methods are used, according to three different environments (RMa, UMa, $\mathrm{UMi}$ ) and two different path loss types (LOS and NLOS). For calculating the BS antenna gain, three different antenna arrays are distinguished, including $4 \times 4,8 \times 8,16 \times 16$.

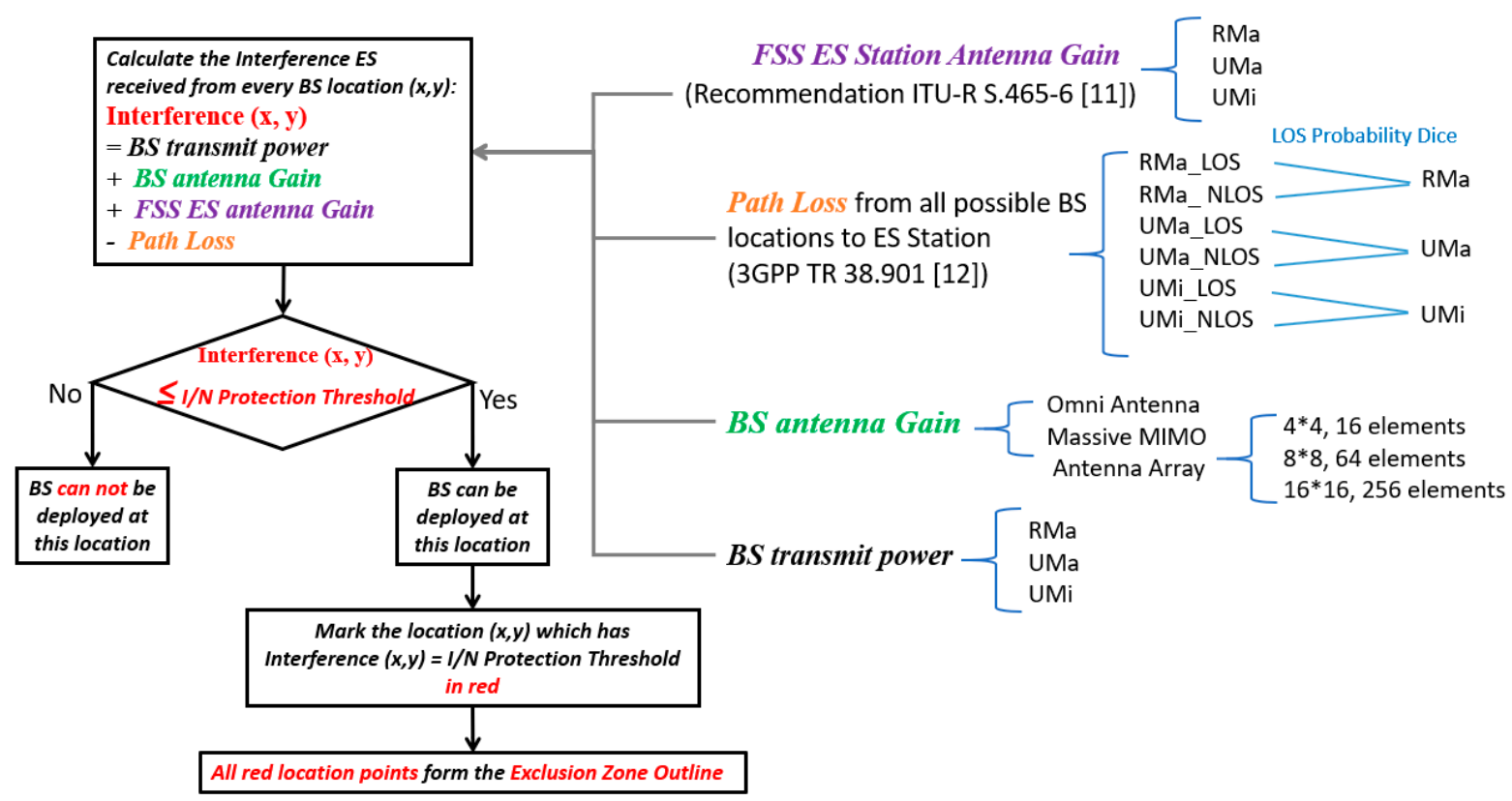

Figure 7. Flowchart of the distance protection method: Exclusion zone. 


\subsection{Restriction Zone and Interactive BS Tx Power Control Scheme}

Up to this point, we have considered the distance protection method, using the exclusion zone to protect the FSS ES from interference from a single BS, where the value of $\mathrm{I} / \mathrm{N}$ is less than, or equal to, the threshold. Outside the exclusion zone, the interference from a single BS is lower than the threshold; however, the aggregate interference from the other BSs exceeds the threshold. Therefore, a restriction zone is introduced to manage the aggregate interference from the cellular network to within the threshold. The derivation of the restriction zone and Tx power control list are as shown in Figures 8 and 9, respectively. Based on the exclusion zone, we consider different target areas to deploy cellular networks, as shown in Figure 8. Then, we calculate the number of BSs in the restricted area, so the interference margin $(-12.2 \mathrm{~dB})$ can then be evenly allocated to the BS outside the restricted area to derive the restriction zone outline. Subsequently, according to the relationship between interference $(x, y)$ and the restriction zone threshold, two kinds of BS Lists are used in the Tx power control inside the restriction zone.

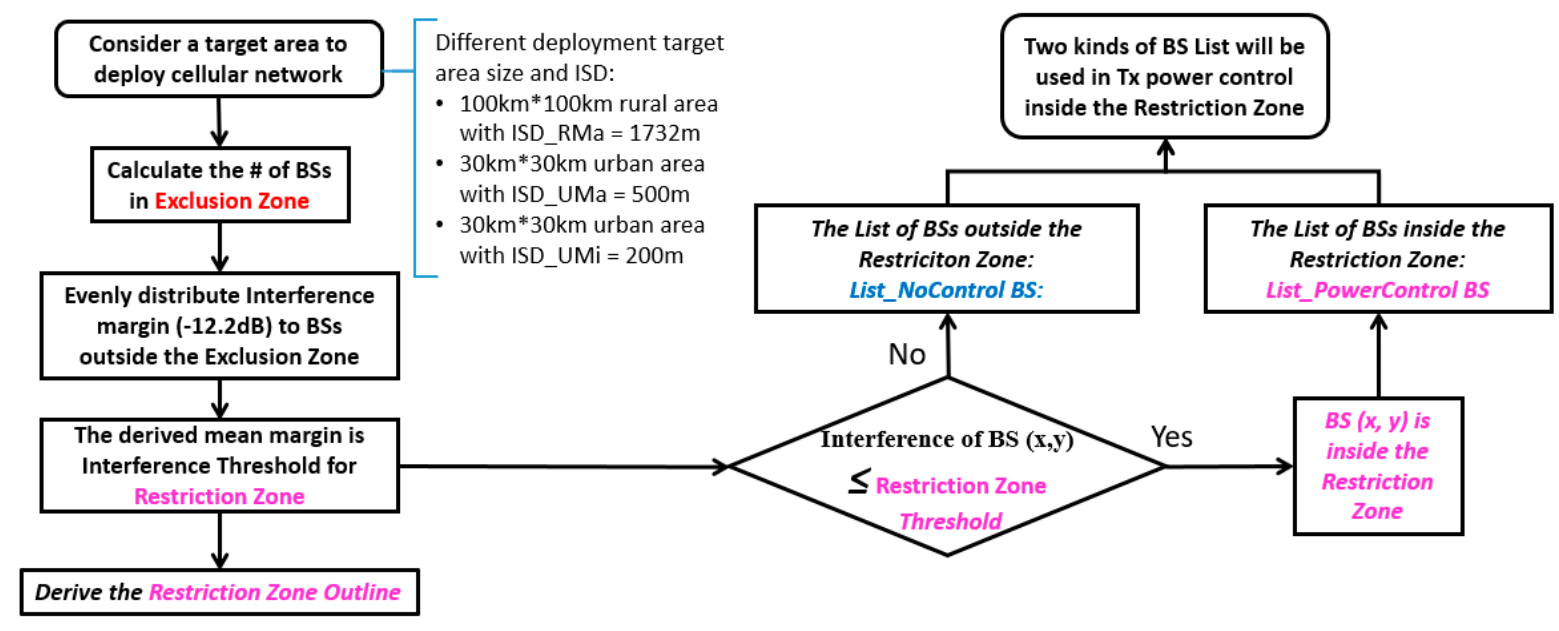

Figure 8. Flowchart for the distance protection method: Restriction zone.

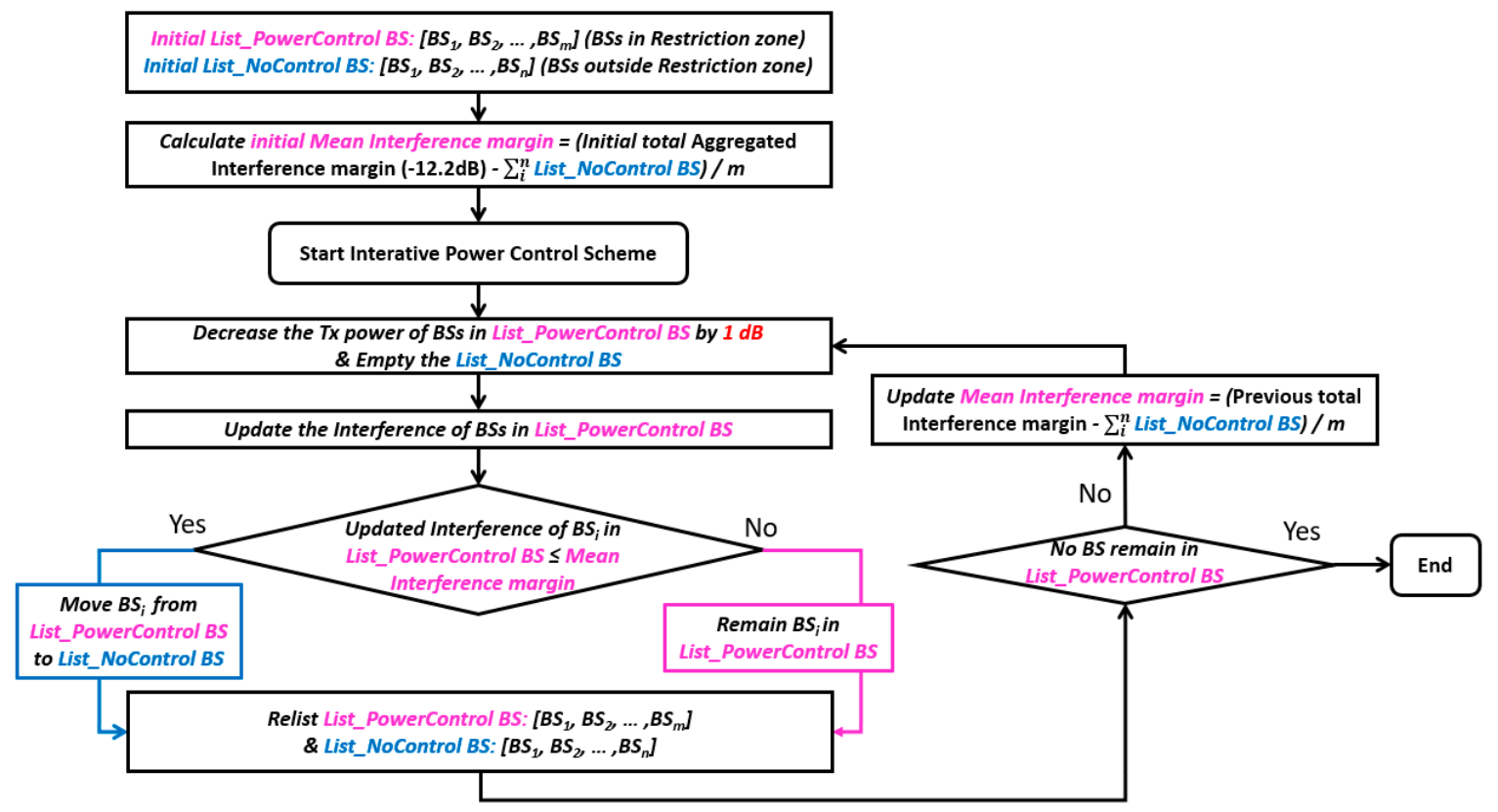

Figure 9. Flowchart for distance protection method: Interactive BS Tx power control scheme. 
To control the Tx power of the BS, so that the total interference of all BASs is lower than the interference margin, this paper proposes the Iterative BS Tx Power Control Scheme inside the restriction zone, as shown in Figure 9. Iterative BS Tx Power Control Scheme inside the restriction zone is based on the previous method and determines whether to start Interactive Power Control Scheme by calculating the initial mean interference margin. After starting the scheme, first, we reduce the Tx power of the BS in the List_PowerControl BS by $1 \mathrm{~dB}$ and clear the List_NoControl BS, to achieve the effect of updating the interference of the $B S_{s}$ in the List_PowerControl BS. If the interference of $B S_{i}$ in the updated List_PowerControl BS is less than, or equal to, the mean interference margin, it needs to be moved to the List_NoControl BS, otherwise the Remain $B S_{i}$ in List_PowerControl BS. If no BS remains in the List_PowerControl BS after relisting, the procedure ends. Otherwise, we need to update the mean interference margin, and then continue with the previous steps, knowing that there is no BS left. Compared with the restriction zone of the simple uniform BS Tx power control scheme, the advantage of the iterative power control scheme is its fairness. In this scheme, the BSs that create relatively lower interferences can be removed from the power control earlier, which results in lower Tx power reduction. Additionally, the BSs that generate relatively higher interferences remain in the power control list for an additional iteration of power reduction.

\section{Numerical Results}

\subsection{Exclusion Zone}

\subsubsection{The Exclusion Zone When Using Omni BS Antenna}

Herein, we investigate the exclusion zone with an omnidirectional BS antenna. In the RMa scenario, the $\mathrm{x}$ and $\mathrm{y}$ axes are the BS location coordinates, and the $\mathrm{z}$ axis is the I/N value, for which we consider the interference from a single BS. No BS can be deployed in the exclusion zone. The randomness of the LOS/NLOS path losses results in random behavior of the I/N value. For instance, the diagrams below show the case for RMa, where the BS location points at which $\mathrm{I} / \mathrm{N}>-12.2 \mathrm{~dB}$ are marked in red in Figure 10. The exclusion zone, in this case, is the area within the outline. Since the main lobe of the ES antenna faces true north, a higher gain results in higher interference from the BS. Hence, the exclusion zone has a larger coverage area on the north side of the ES. Owing to the randomness of the LOS/NLOS path losses and the I/N value in the UMa scenario also demonstrates random behavior; therefore, the points with $-10 \mathrm{~dB}$ I/ $\mathrm{N}$ are no longer located on a perfect circle. The exclusion zone is the area where no $-10 \mathrm{~dB}$ points (marked in red) exist, as shown in Figure 11. For the UMi scenario, the exclusion zone is smaller than that of the UMa; this is because the antenna height difference between the BS and ES is larger. The larger antenna height difference, the longer the propagation distance, which causes higher path loss. This results in lower interference and a smaller exclusion zone, as shown in Figure 12.

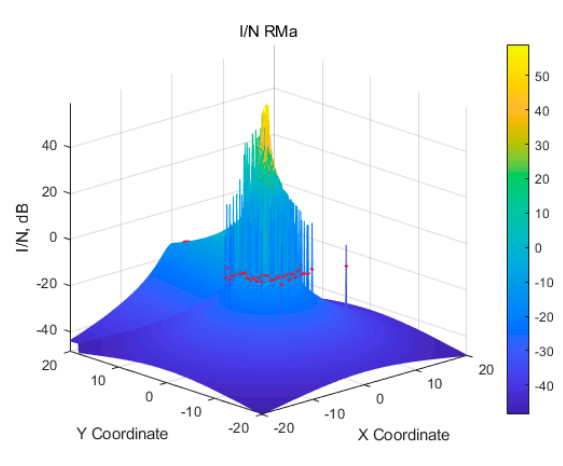

(a) 3D Version of RMa scenario

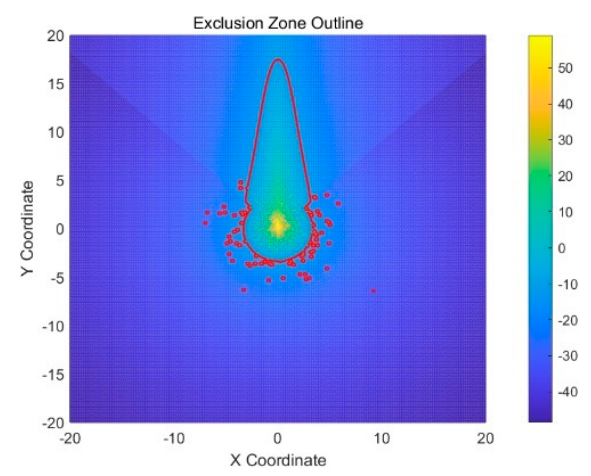

(b) Top View (2D) of RMa scenario

Figure 10. RMa scenario with ES antenna elevation angle of $5^{\circ}$. 


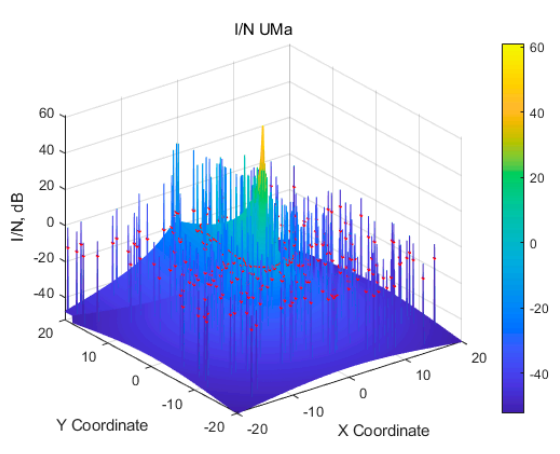

(a) 3D Version of UMa scenario

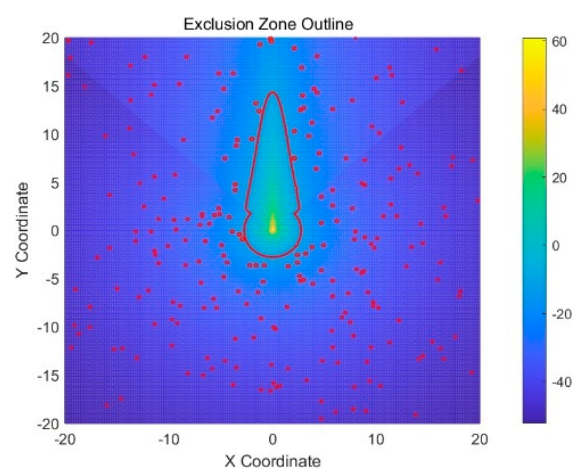

(b) Top View (2D) of UMa scenario

Figure 11. UMa scenario with ES antenna elevation angle of $5^{\circ}$.

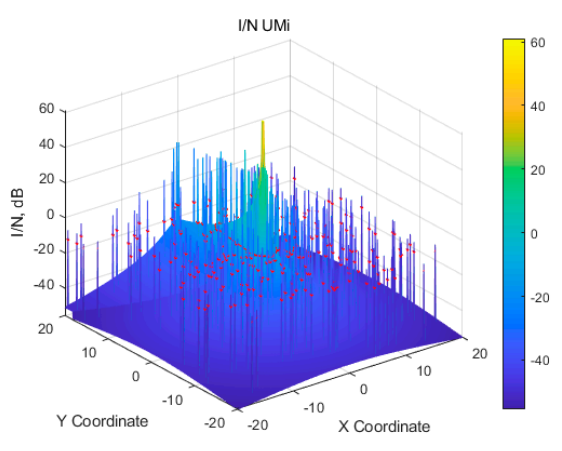

(a) 3D Version of UMi scenario

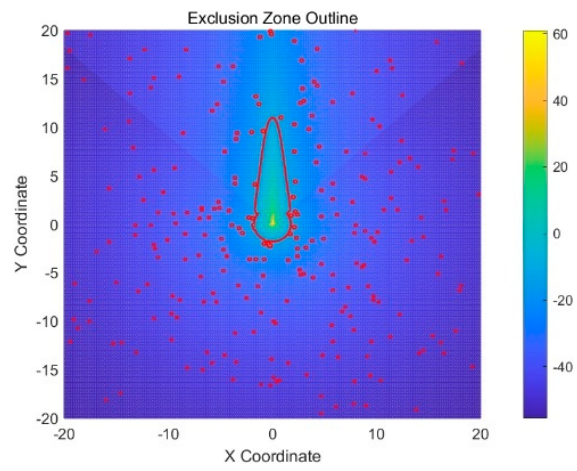

(b) Top View (2D) of UMi scenario

Figure 12. UMi scenario with ES antenna elevation angle of $5^{\circ}$.

\subsubsection{The Exclusion Zone When Using Beamforming Antenna Array}

The omnidirectional antenna provides a maximum gain in all directions. Herein, we consider a massive MIMO antenna array with beamforming. For the coexistence study, the worst interference case is considered for both the horizontal and vertical domains.

- Azimuth plane:

By electronic steering, a planar URA can generate the main lobe beam, which can sweep in all horizontal directions. For each BS, the interference from every beam direction $\left(360^{\circ}\right)$ is checked, and the maximum interference is considered as the BS interference.

- Elevation plane:

The beam angle changes with the user location, and the worst case is obtained for a user at the cell edge. Such a user has the smallest beam off-boresight angle (Figure 13).

Having a larger number of elements in the antenna array enables a higher gain for the main-lobe beam. This increases the interference from the BS at the ES, thus resulting in a larger exclusion zone. Using the beamforming antenna array, the exclusion zone becomes larger, owing to the increased number of antenna elements. Figures 14-17 show the exclusion zones in the presence of the BS beamforming antenna array for the RMa, UMa, and UMi scenarios. 


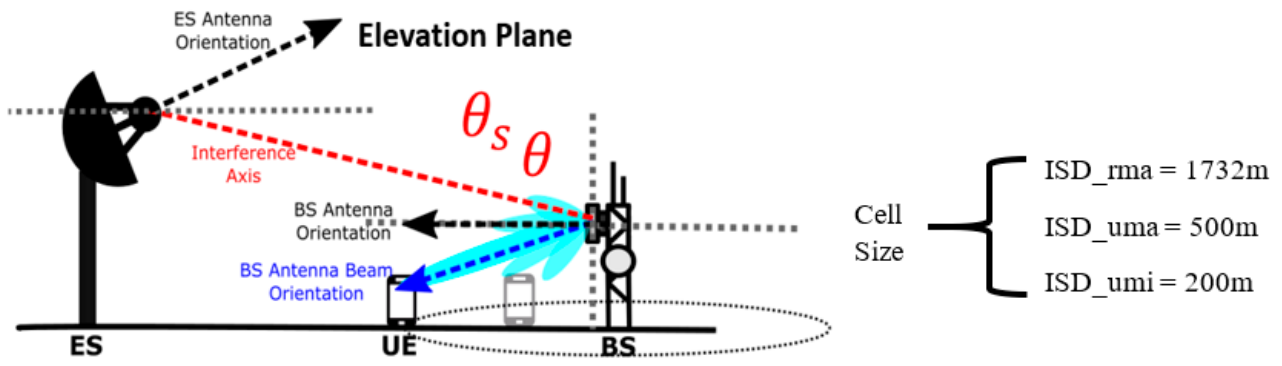

Figure 13. The elevation plane.
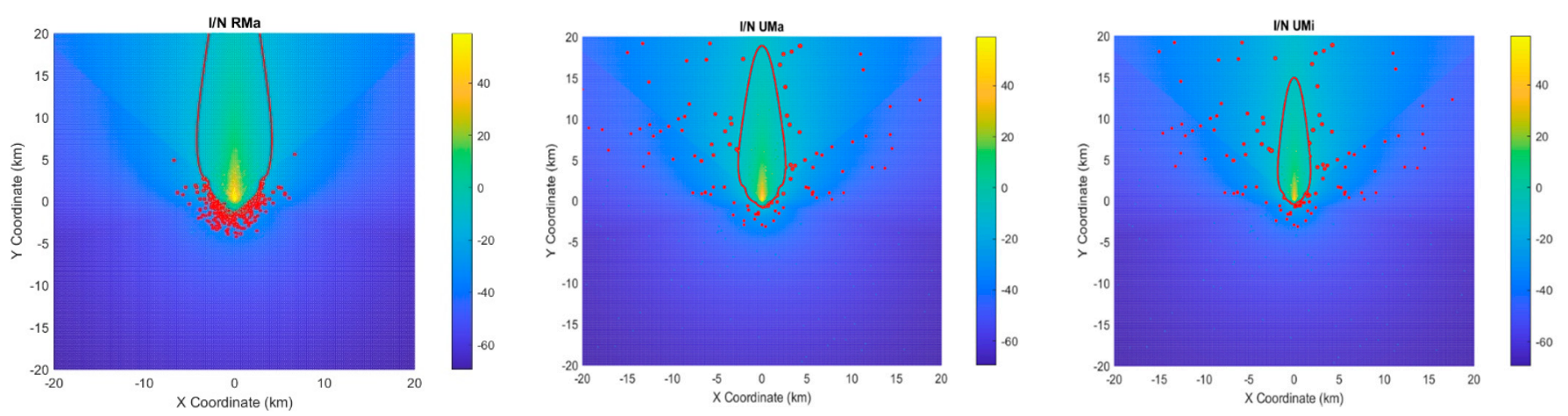

Figure 14. RMa, UMa, and UMi scenario with an ES antenna elevation angle of $5^{\circ}$ for a $4 \times 4$ beamforming antenna array.
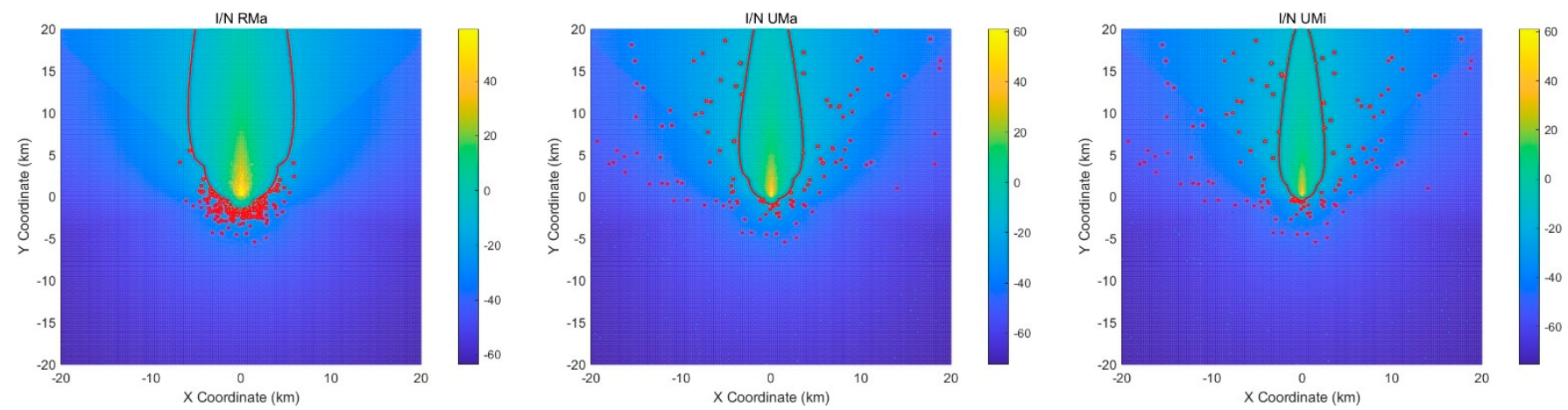

Figure 15. RMa, UMa, and UMi scenarios with ES antenna elevation angle of $5^{\circ}$ for an $8 \times 8$ beamforming antenna array.
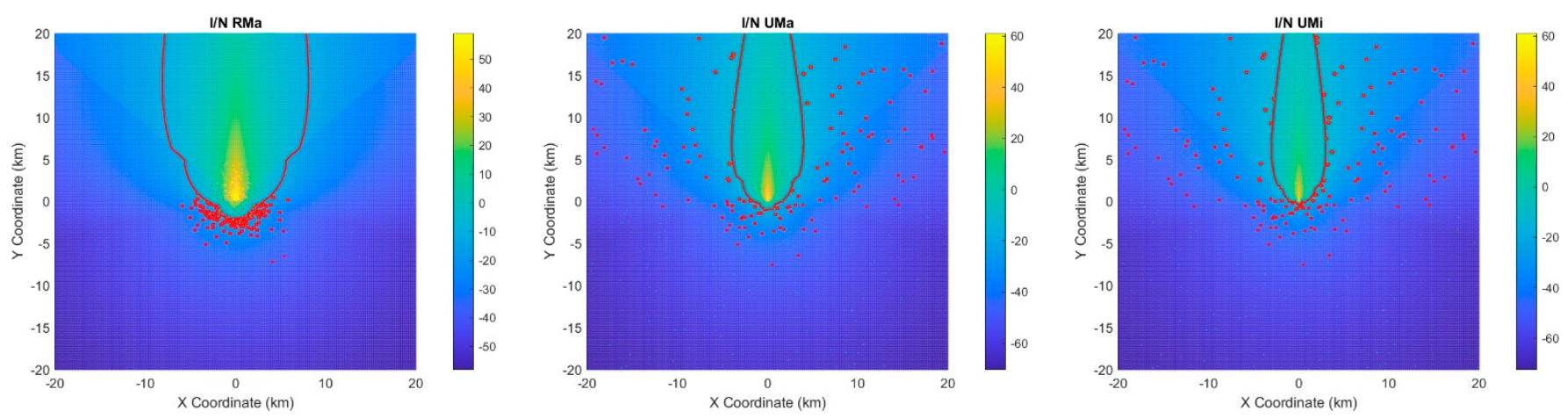

Figure 16. RMa, UMa, and UMi scenarios with ES antenna elevation angle of $5^{\circ}$ for a $16 \times 16$ beamforming antenna array. 

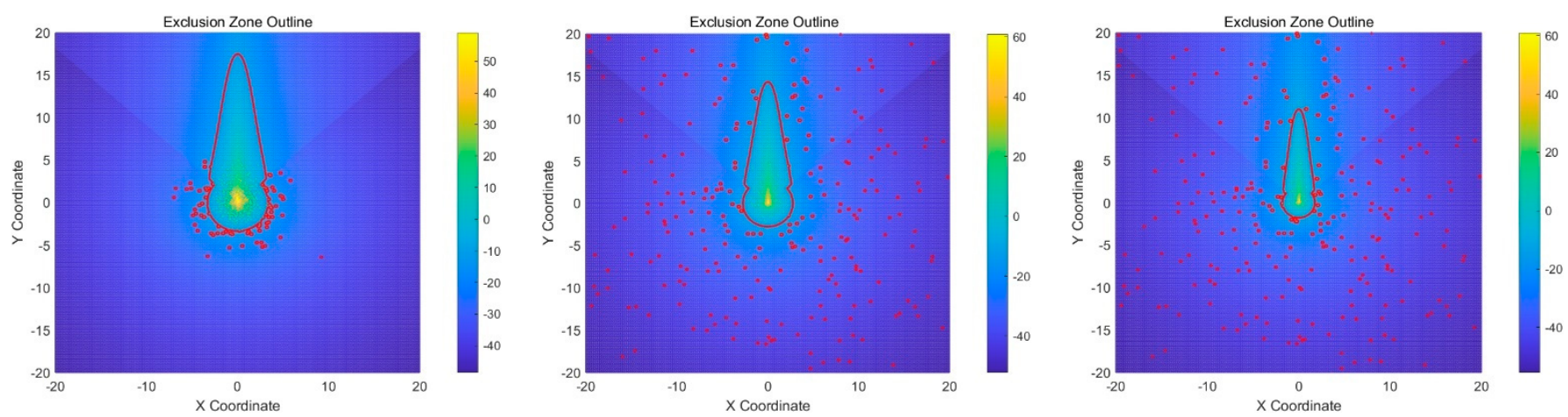

Figure 17. RMa, UMa, and UMi scenarios with an ES antenna elevation angle of $5^{\circ}$ for the conventional omnidirectional antenna.

Comparing the results in Figure 14 with those in Figure 17, it is observed that the exclusion zone is larger at the north side of the ES when the antenna array is used. This is because the maximum gain of the antenna array is higher than that of the omnidirectional antenna, i.e., $\max 20 \mathrm{~dB}$ of a $4 \times 4$ array $>15 \mathrm{~dB}$ of the omnidirectional antenna. On the south side of the ES, however, the exclusion zone is much smaller in the case of the antenna array; this is attributed to the directional attenuation pattern of the antenna array. Comparing the results in Figures 14-16, It can be found that the exclusion zone becomes larger when the number of elements increases. Because more elements in antenna array leads to higher antenna array gain of main lobe beam, higher interference from BS to ES, and bigger exclusion zone.

\subsubsection{The Effects of ES Elevation Angle on Exclusion Zone}

As discussed above, the ES elevation angle has a significant effect on the ES antenna gain, which greatly affects the exclusion zone. Depending on the location of the satellite, the ES antenna may have different tilt angles [3]. It is reported that the elevation angle generally varies from 5-48 [13]. Figures 18-21 demonstrate the effects of the elevation angle of the ES antenna in the exclusion zone for two typical angles, $15^{\circ}$ and $48^{\circ}$, respectively, as given in [13]. Note that, as the elevation angle increases, the off-boresight angle also increases. This results in lower ES antenna gain toward the north side, causing lower interference and hence a smaller exclusion zone.
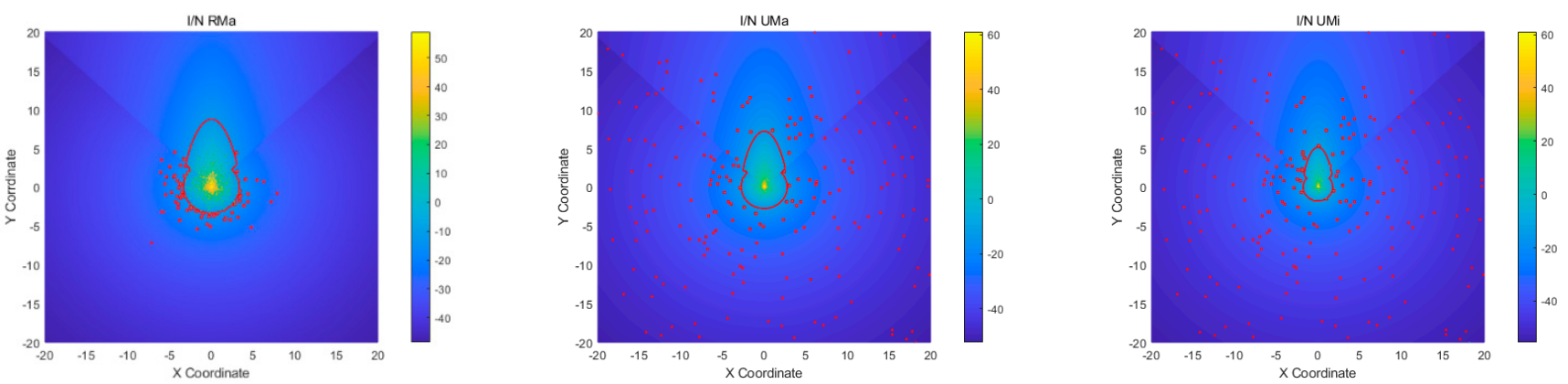

Figure 18. RMa, UMa, and UMi scenarios with an ES antenna elevation angle of $15^{\circ}$ for the conventional omnidirectional antenna. 

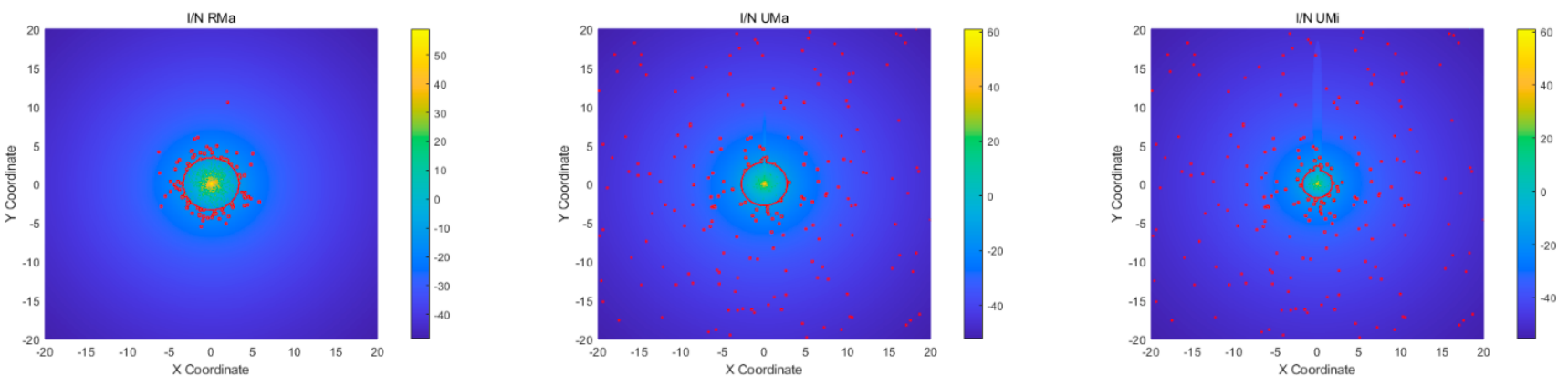

Figure 19. RMa, UMa, and UMi scenarios with an ES antenna elevation angle of $48^{\circ}$ for the conventional omnidirectional antenna.
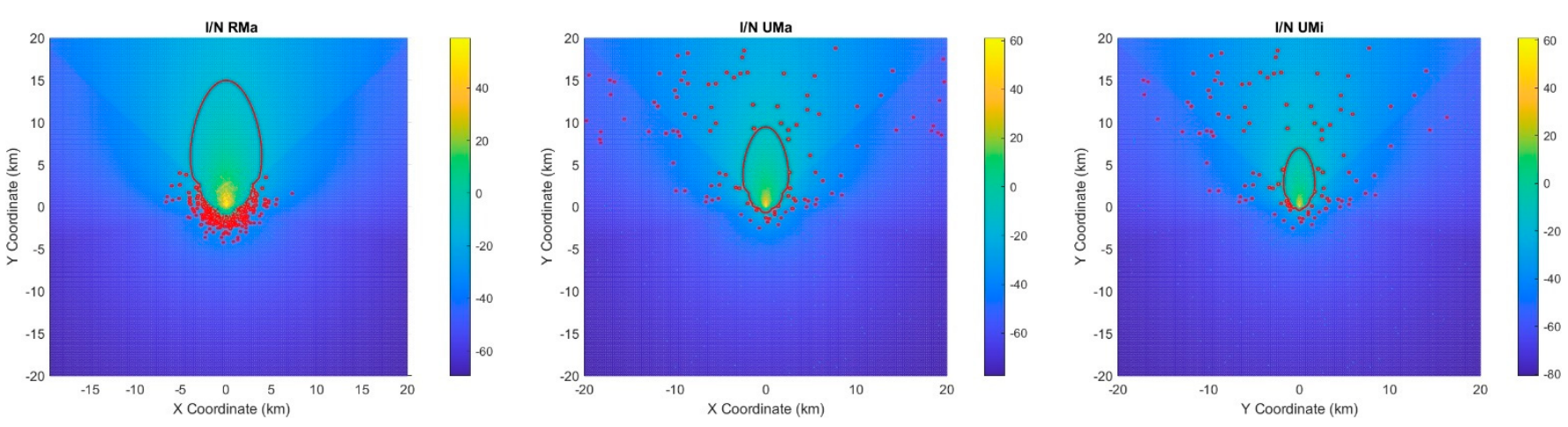

Figure 20. RMa, UMa, and UMi scenarios with an ES antenna elevation angle of $15^{\circ}$ for a $4 \times 4$ antenna array.
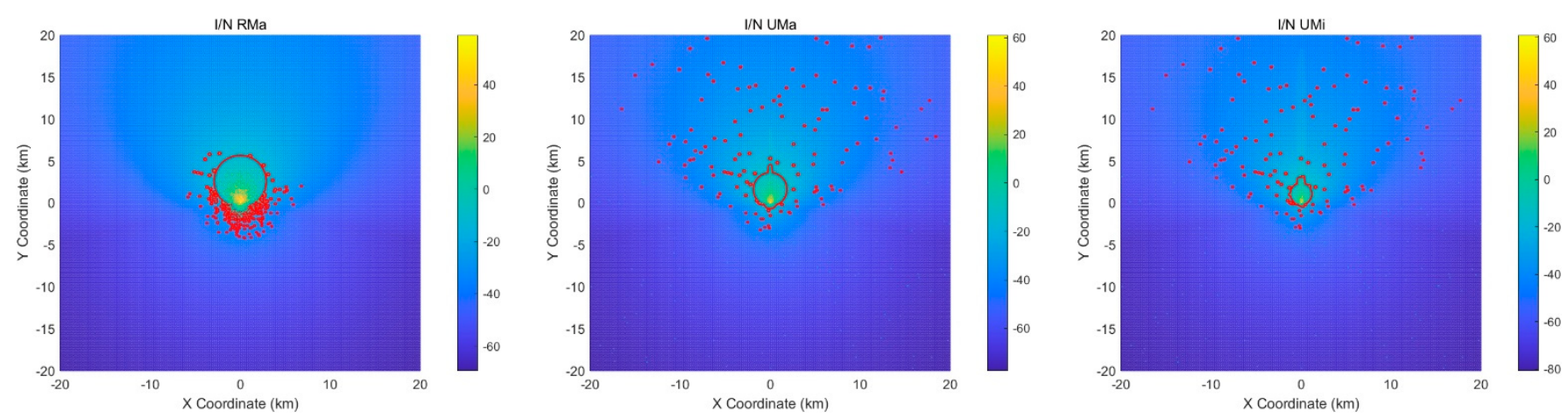

Figure 21. RMa, UMa, and UMi scenarios with an ES antenna elevation angle of $48^{\circ}$ for a $4 \times 4$ antenna array.

\subsection{Restriction Zone}

\subsubsection{Restriction Zone and BS Tx Power Control (Omni Antenna Results)}

Figure 22 shows the sizes of the exclusion and restriction zones. For the UMa and UMi cases, all the BSs located outside the exclusion zone require Tx power control. As illustrated in Figure 22, in the RMa, most of the BSs only need to decrease their Tx power by $1 \mathrm{~dB}$; however, most of the BSs in the UMa must decrease their Tx power by $5-10 \mathrm{~dB}$, and most of the BSs in the UMi must decrease their Tx power by 10-15 $\mathrm{dB}$. The UMi requires the highest mean Tx power reduction because it has the smallest ISD, which indicates a larger number of BSs in the area. Figure 23 also shows the BS Tx power reduction against the number of BSs, i.e., nearly 390 BSs need to only decrease their Tx power by $1 \mathrm{~dB}$ in the RMa scenario. 

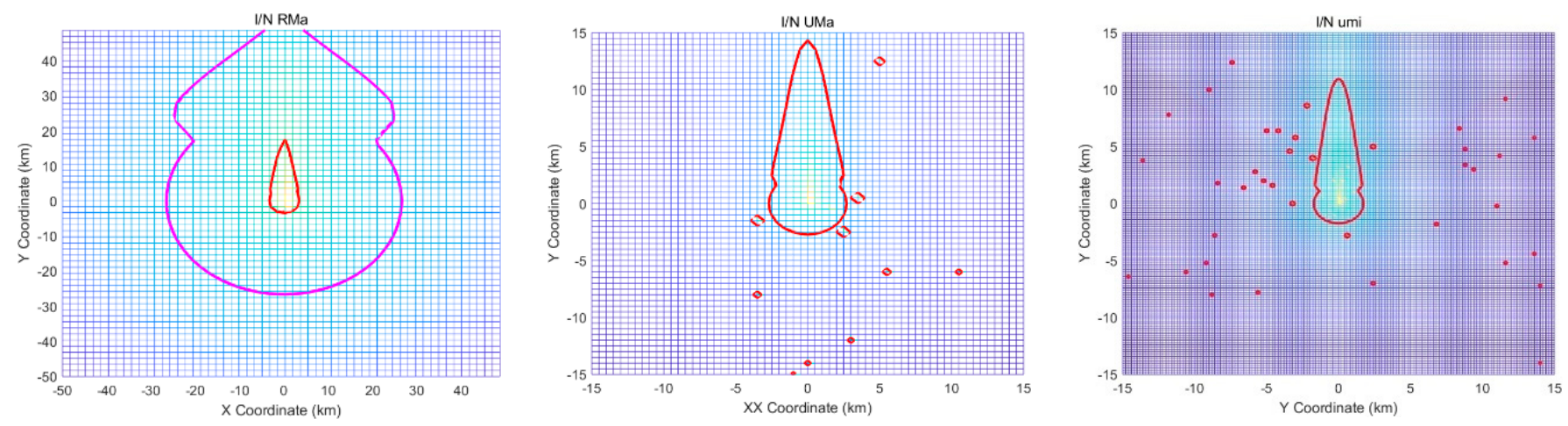

Figure 22. The size of the exclusion and restriction zones for different scenarios using omni antenna. The red lines show the exclusion zone (no BSs can be deployed) and the pink lines show the restriction zone (BSs can be deployed with Tx power control). The blue lines indicate that BSs can be deployed in these areas without restrictions.
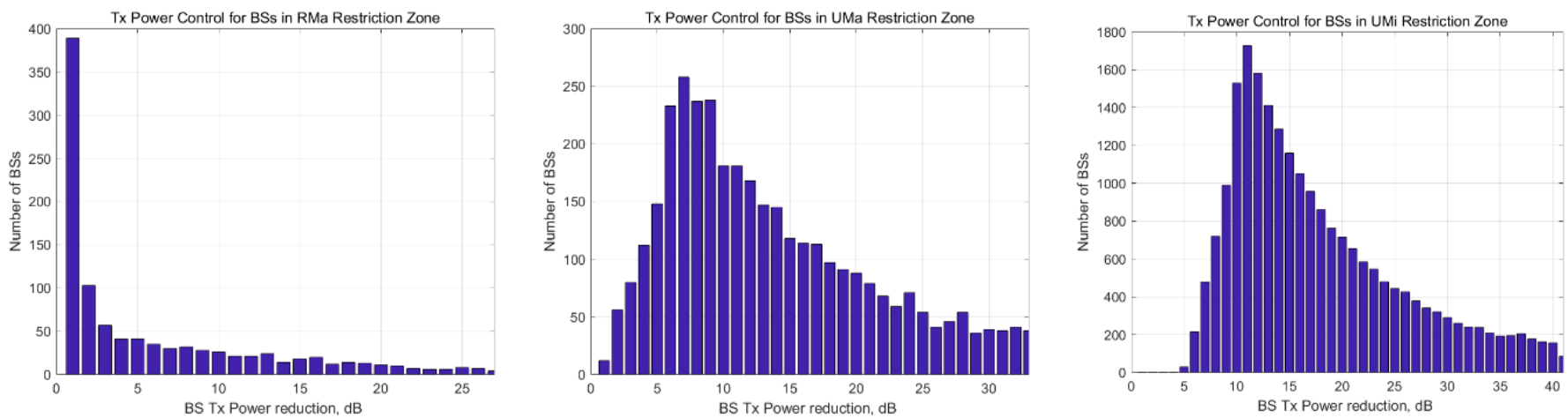

Figure 23. Mean Tx power reduction for the BS versus the number of BSs.

- $\quad \mathrm{RMa}, \mathrm{ISD}=1732 \mathrm{~m}$, in $100 \times 100 \mathrm{~km}$ area:

(1) About 3337 BSs can be deployed outside the exclusion zone in the RMa

(2) The interference threshold for the restriction zone in the RMa is $-47.43 \mathrm{~dB}$

(3) About 998 BSs within the restriction zone require Tx power control in the RMa

- $\quad \mathrm{UMa}, \mathrm{ISD}=500 \mathrm{~m}$, in $30 \times 30 \mathrm{~km}$ area:

(1) About 3484 BSs can be deployed outside the exclusion zone in the UMa

(2) The interference threshold for the restriction zone in the UMa is $-47.62 \mathrm{~dB}$

(3) About 3484 BSs within the restriction zone require Tx power control in the UMa

- UMi, ISD = $200 \mathrm{~m}$, in $30 \times 30 \mathrm{~km}$ area:

(1) About 22,061 BSs can be deployed outside the exclusion zone in the UMi

(2) The interference threshold for the restriction zone in the UMi is $-55.64 \mathrm{~dB}$

(3) About 22,061 BSs within the restriction zone need Tx power control in the UMi

\subsubsection{Restriction Zone and BS Tx Power Control for $4 \times 4$ Antenna Array}

It is seen from Figures 24 and 25 that, although the exclusion zone is larger for the BSs than using the omnidirectional antenna, the restriction zone is much smaller, especially in the rear of the ES antenna. This is owed to the directional attenuation of the MIMO antenna array. The UMi has the largest restriction zone, although it has the smallest exclusion zone; furthermore, its required mean Tx power reduction is highest because it has the smallest ISD.

- $\quad \mathrm{RMa}, \mathrm{ISD}=1732 \mathrm{~m}$, in $100 \times 100 \mathrm{~km}$ area:

(1) About 3320 BSs can be deployed outside the exclusion zone in the RMa

(2) The interference threshold for the restriction zone in the RMa is $-47.39 \mathrm{~dB}$ 
(3) About 606 BSs within the restriction zone require Tx power control in the RMa

- $\quad \mathrm{UMa}, \mathrm{ISD}=500 \mathrm{~m}$, in $30 \times 30 \mathrm{~km}$ area:

(1) About 3463 BSs can be deployed outside the exclusion zone in the UMa

(2) The interference threshold for the restriction zone in the UMa is $-47.59 \mathrm{~dB}$

(3) About 1450 BSs within the restriction zone require Tx power control in the UMa

- $\mathrm{UMi}, \mathrm{ISD}=200 \mathrm{~m}$, in $30 \times 30 \mathrm{~km}$ area:

(1) About 21,831 BSs can be deployed outside the exclusion zone in UMi

(2) The interference threshold for the restriction zone in the UMi is $-55.59 \mathrm{~dB}$

(3) About 10,960 BSs within the restriction zone require Tx power control in the UMi.

Unlike the cases with the omnidirectional antennas, most of the BSs need to decrease their Tx power by $1 \mathrm{~dB}$, not only in the RMa but also in the UMa and UMi. This is because the $4 \times 4$ antenna has a higher gain than the omnidirectional antenna but has a similar mean power reduction value. This means that a lower power reduction is more efficient in the UMa and UMi using the $4 \times 4$ antenna array. However, the UMi still requires the highest mean Tx power reduction.
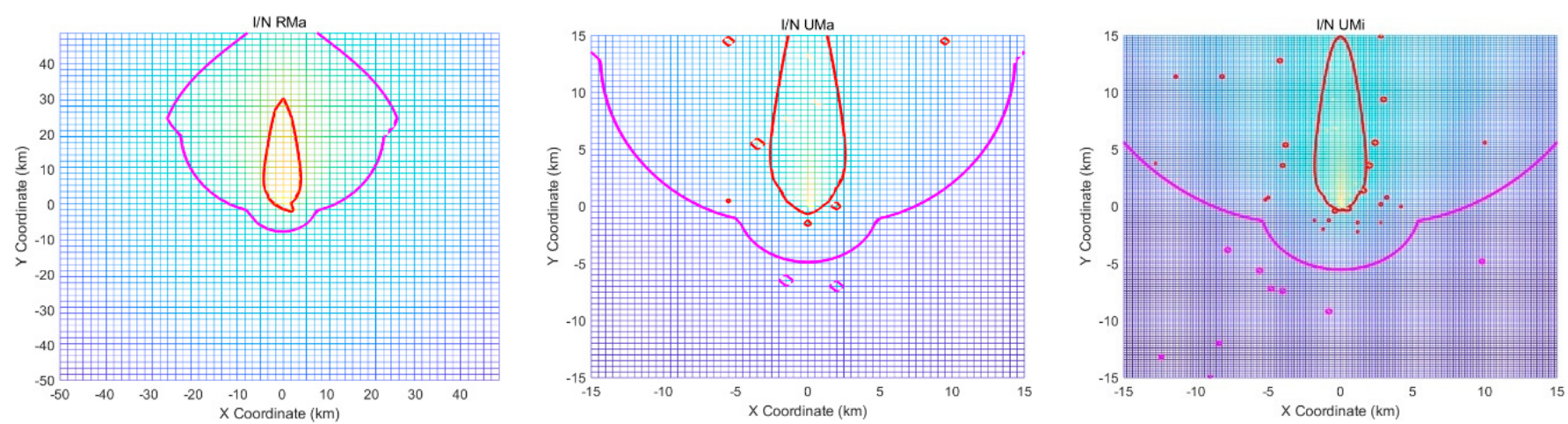

Figure 24. The size of the exclusion and restriction zones for different scenarios using $4 \times 4$ antenna array.
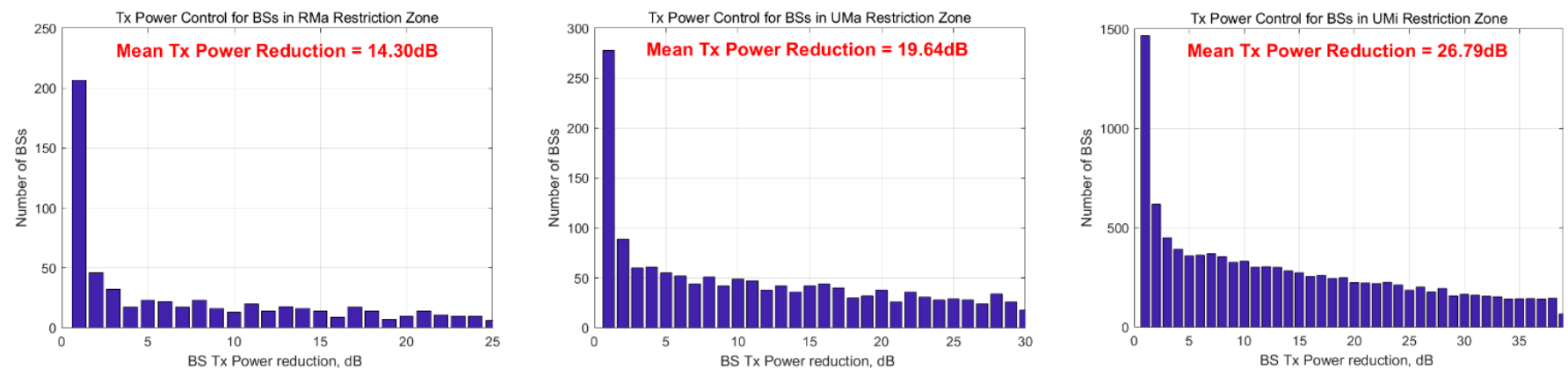

Figure 25. Mean Tx power reduction for the BS versus the number of BSs.

In Figures 26-31, we further compare the results using different antennas.

(a) For the RMa scenario, the array antenna has a higher gain and larger exclusion zone. Although the exclusion zone is larger than that of the omnidirectional antenna, the rear restriction zone of the ES antenna is much smaller. This is because of the directional attenuation of the MIMO antenna array. The larger the number of elements in the array, the larger the exclusion zone. This also results in a larger restriction zone, owing to the higher gain of the antenna array, as shown in Figure 26. 

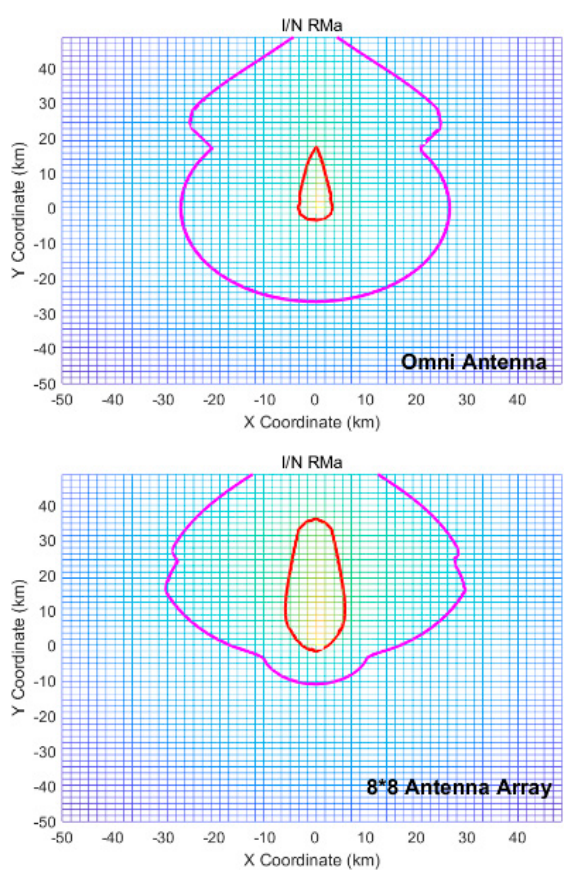
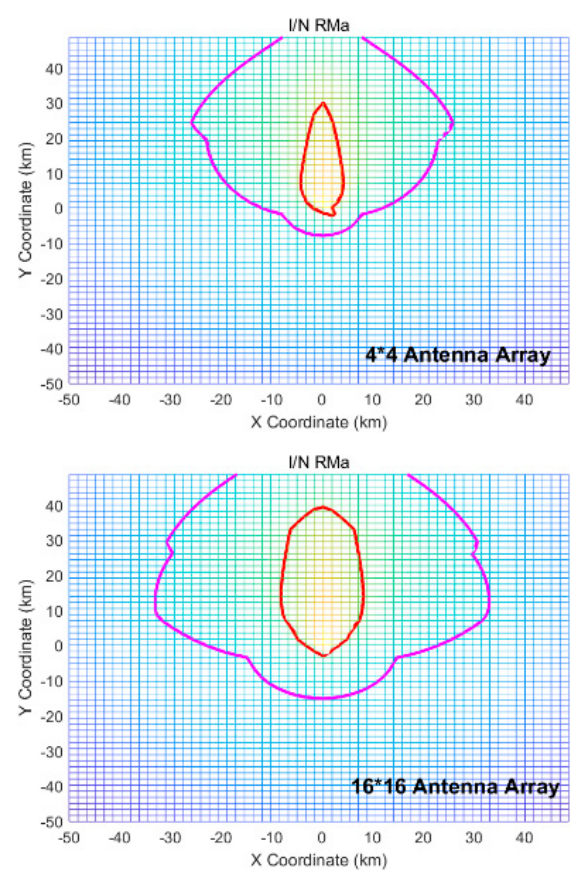

Figure 26. The size of exclusion and restriction zones for different RMa scenarios.

The following results are seen from Figure 27 and Table 4:

- Because of the larger exclusion zone, the number of BSs that can be deployed continues to decrease with the increasing number of array elements.

- Comparing the omnidirectional antenna and $4 \times 4$ antenna array, the number, and percentage of BSs that require Tx power control are significantly lower because of the drastic shrinkage of the restriction zone. However, owing to the higher antenna gain, the mean Tx power reduction is higher for the case of BSs with a $4 \times 4$ antenna array.

- By increasing the number of antenna elements, the number and percentage of BSs requiring Tx power control also increase, owing to the expanded restriction zone.

- By increasing the number of antenna elements, the maximum Tx power and mean Tx power reduction increase, owing to the higher antenna gain.

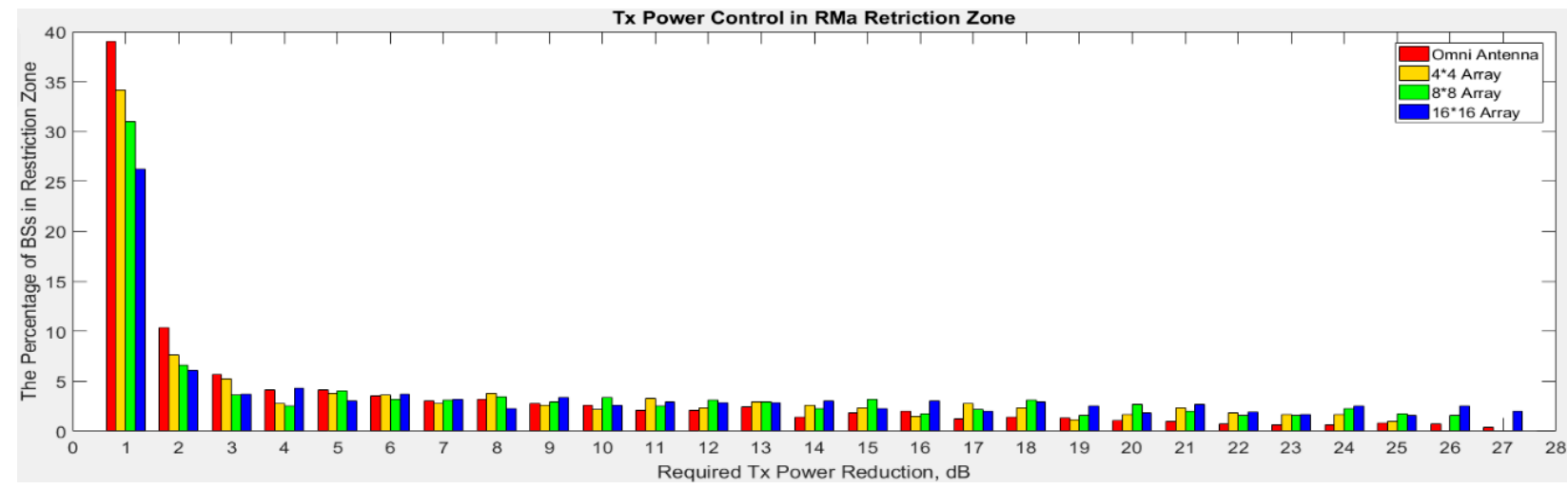

Figure 27. Tx power control chart in the RMa restriction zone. 
Table 4. Mean Tx power reduction in the restriction zone and the BS Tx power control in the RMa.

\begin{tabular}{cccccc}
\hline Antenna Type & $\begin{array}{c}\text { Total \# of BSs } \\
\text { That Can Be } \\
\text { Deployed }\end{array}$ & $\begin{array}{c}\text { Interference } \\
\text { Threshold for } \\
\text { Restriction Zone }\end{array}$ & $\begin{array}{c}\text { Number of BSs in } \\
\text { the Restriction } \\
\text { Zone }\end{array}$ & $\begin{array}{c}\text { \% of Deployed } \\
\text { BSs That Require } \\
\text { Tx Power Control }\end{array}$ & $\begin{array}{c}\text { Mean Tx Power } \\
\text { Reduction in the } \\
\text { Restriction Zone }\end{array}$ \\
\hline Omni & 3337 & $-47.43 \mathrm{~dB}$ & 998 & 29.91 & $13.18 \mathrm{~dB}$ \\
$4 \times 4$ Array & 3302 & $-47.39 \mathrm{~dB}$ & 606 & 18.35 & $14.30 \mathrm{~dB}$ \\
$8 \times 8$ Array & 3252 & $-47.32 \mathrm{~dB}$ & 746 & 22.93 & $15.84 \mathrm{~dB}$ \\
$16 \times 16$ Array & 3194 & $-47.24 \mathrm{~dB}$ & 886 & 27.74 & $17.51 \mathrm{~dB}$ \\
\hline
\end{tabular}

(b) For the UMa, the array antenna has a higher gain, thus causing a larger exclusion zone. Although the exclusion zone is larger than that of the omnidirectional antenna, the restriction zone at the backside of the ES antenna is much smaller. This is owed to the directional attenuation of the MIMO antenna array. As there are larger numbers of elements in the arrays, the exclusion and restriction zones are larger because of the higher antenna array gains, as shown in Figure 28.

Unlike the RMa, as shown in Figure 29 and Table 5, the mean Tx power reduction is lower when the antenna array is used. As seen from the histogram, when using the antenna array, many of the cells only need to reduce their Tx power by $1 \mathrm{~dB}$. In contrast, when using an omnidirectional antenna, a $1 \mathrm{~dB}$ power reduction barely makes any difference, and most of the BSs need to decrease their Tx power by $6-12 \mathrm{~dB}$. Therefore, the proposed power control scheme is more efficient when using an antenna array in UMa.
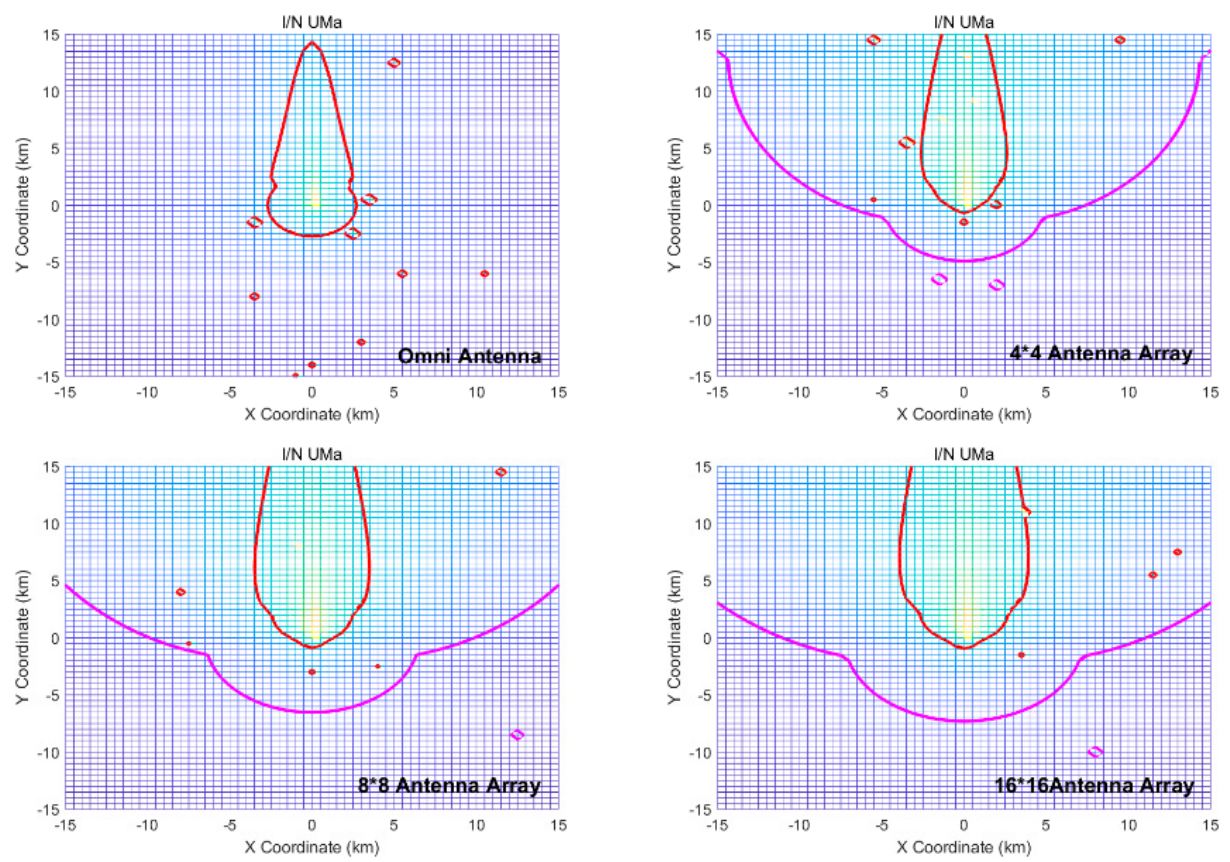

Figure 28. Sizes of exclusion and restriction zones for various UMa scenarios. 


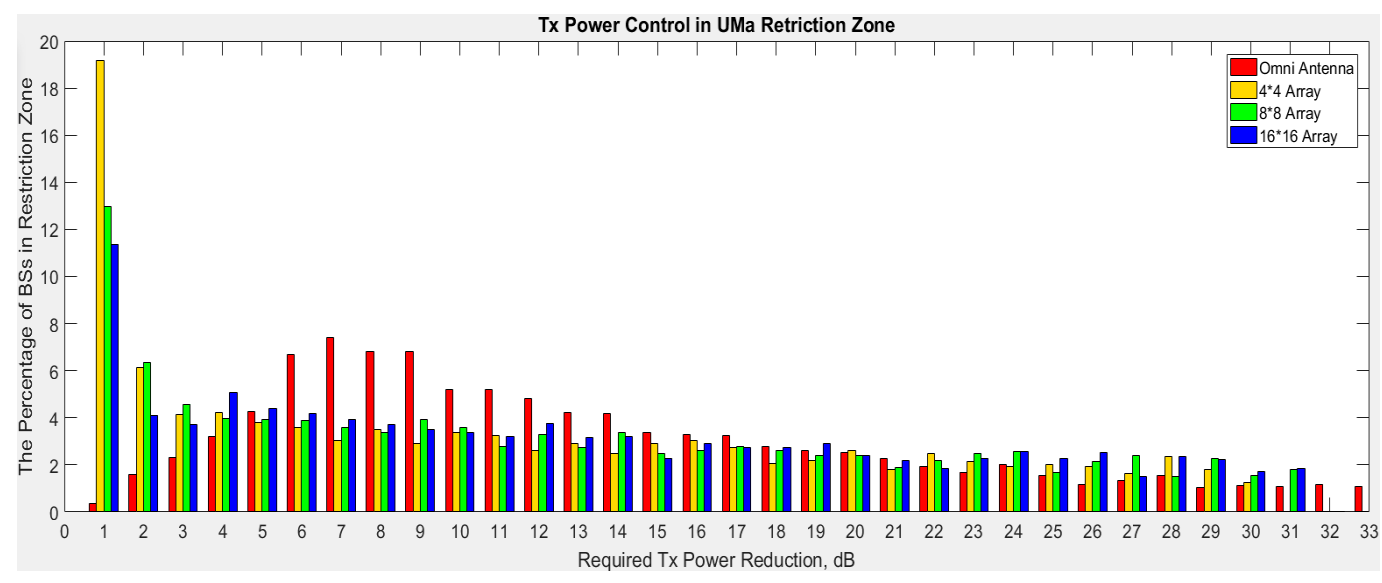

Figure 29. Tx power control chart in UMa restriction zone.

Table 5. Mean Tx power reduction in the restriction zone and the BS Tx power control in Uma.

\begin{tabular}{cccccc}
\hline Antenna Type & $\begin{array}{c}\text { Total \# of BSs } \\
\text { That Can Be } \\
\text { Deployed }\end{array}$ & $\begin{array}{c}\text { Interference } \\
\text { Threshold for } \\
\text { Restriction Zone }\end{array}$ & $\begin{array}{c}\text { Number of BSs in } \\
\text { the Restriction } \\
\text { Zone }\end{array}$ & $\begin{array}{c}\text { \% of Deployed } \\
\text { BSs That Require } \\
\text { Tx Power Control }\end{array}$ & $\begin{array}{c}\text { Mean Tx Power } \\
\text { Reduction in the } \\
\text { Restriction Zone }\end{array}$ \\
\hline Omni & 3484 & $-47.62 \mathrm{~dB}$ & 3484 & 100 & $27.05 \mathrm{~dB}$ \\
$4 \times 4$ Array & 3463 & $-47.59 \mathrm{~dB}$ & 1450 & 41.87 & $19.64 \mathrm{~dB}$ \\
$8 \times 8$ Array & 3352 & $-47.45 \mathrm{~dB}$ & 1682 & 50.18 & $20.84 \mathrm{~dB}$ \\
$16 \times 16$ Array & 3300 & $-47.39 \mathrm{~dB}$ & 1749 & 53.00 & $21.07 \mathrm{~dB}$ \\
\hline
\end{tabular}

(c) For the UMi scenario, the array antenna has a higher gain, which results in a larger exclusion zone. Although the exclusion zone is larger than that of the omnidirectional antenna, the restriction zone at the rear of the ES antenna is much smaller. This is owed to the directional attenuation of the MIMO antenna array. Note that there are a larger number of elements in the array; therefore, the exclusion and restriction zones are larger because of the higher gain of the antenna array, as shown in Figure 30.
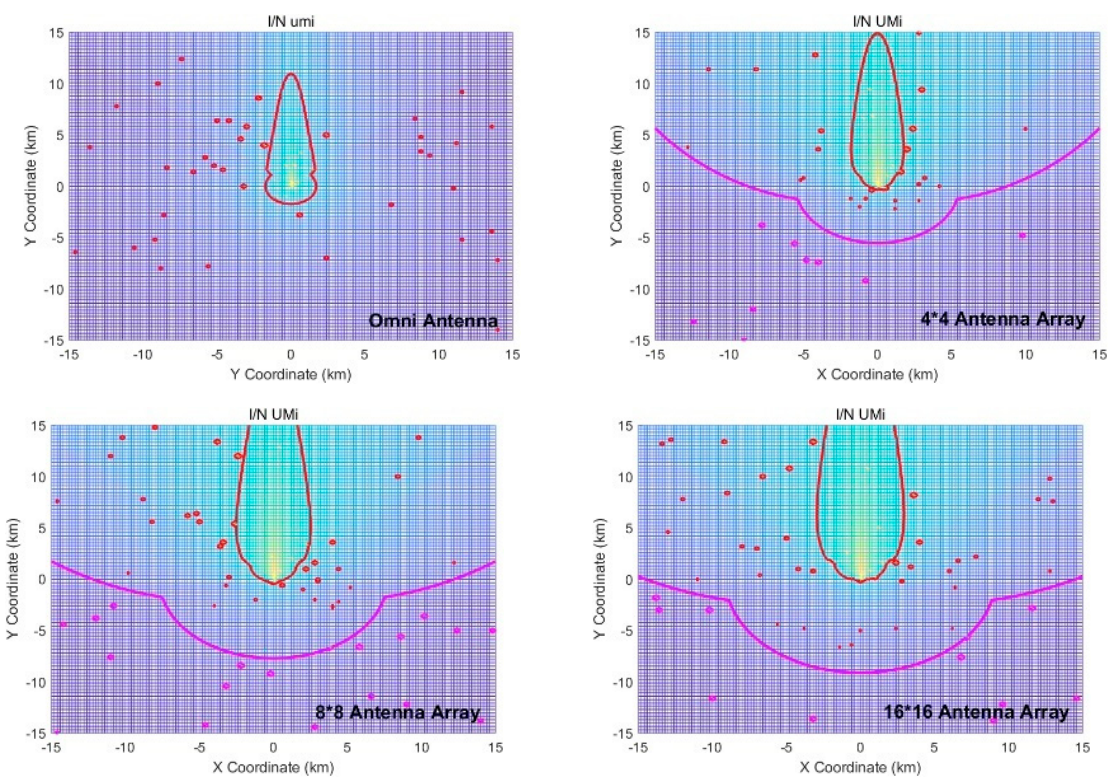

Figure 30. Sizes of the exclusion and restriction zones for UMi. 
As shown in Figure 31 and Table 6, unlike the RMa, and similar to the UMa, the proposed power control scheme is more efficient when using an antenna array in UMi. However, the difference between the mean Tx power reduction is lower compared to UMa, and the mean Tx power reduction is higher than that of the omnidirectional antenna when using a $16 \times 16$ antenna, owing to its higher gain. From the histogram, the proportion of BSs that require power reduction over $29 \mathrm{~dB}$ is higher, when a $16 \times 16$ antenna array is used instead of an omnidirectional antenna.

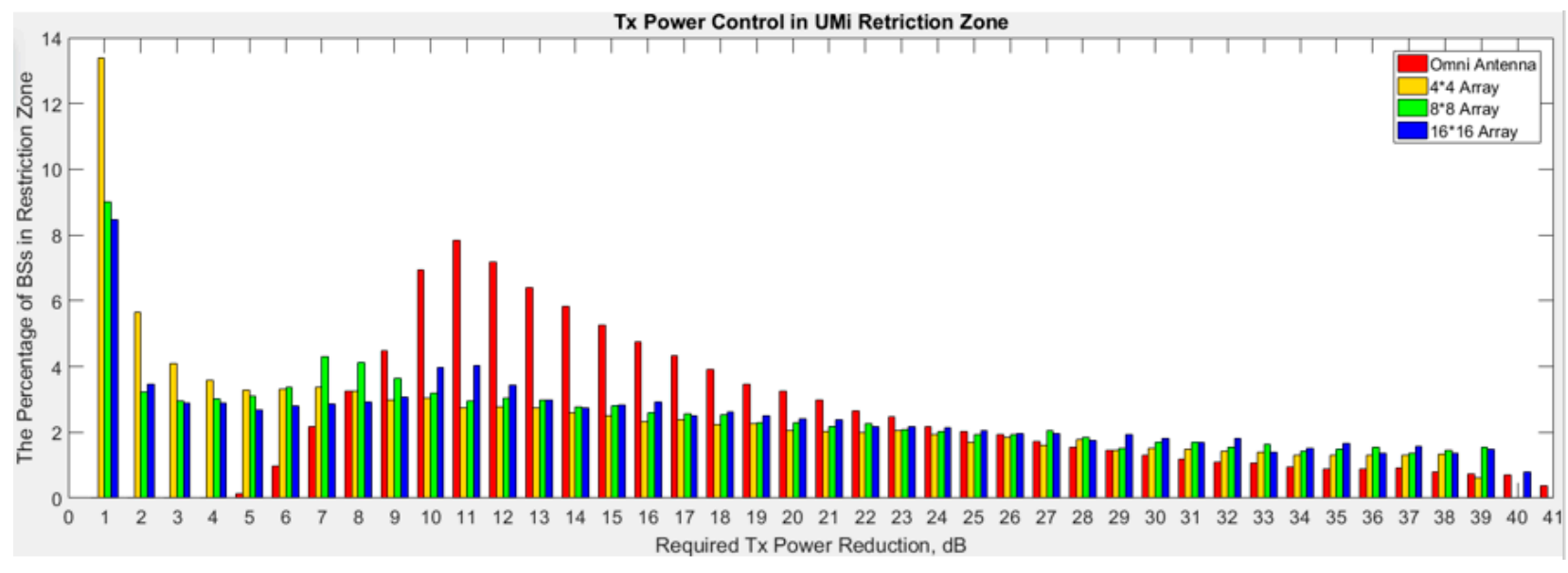

Figure 31. Tx power control chart in UMi scenario.

Table 6. Mean Tx power reduction in the restriction zone and BS Tx power control in UMi.

\begin{tabular}{cccccc}
\hline Antenna Type & $\begin{array}{c}\text { Total \# of BSs } \\
\text { That Can Be } \\
\text { Deployed }\end{array}$ & $\begin{array}{c}\text { Interference } \\
\text { Threshold for } \\
\text { Restriction Zone }\end{array}$ & $\begin{array}{c}\text { Number of BSs in } \\
\text { the Restriction } \\
\text { Zone }\end{array}$ & $\begin{array}{c}\text { \% of Deployed } \\
\text { BSs That Require } \\
\text { Tx Power Control }\end{array}$ & $\begin{array}{c}\text { Mean Tx Power } \\
\text { Reduction in the } \\
\text { Restriction Zone }\end{array}$ \\
\hline Omni & 22,061 & $-55.63 \mathrm{~dB}$ & 22,061 & 100 & $27.05 \mathrm{~dB}$ \\
$4 \times 4$ Array & 21,831 & $-55.59 \mathrm{~dB}$ & 10,960 & 50.20 & $26.79 \mathrm{~dB}$ \\
$8 \times 8$ Array & 21,193 & $-55.46 \mathrm{~dB}$ & 12,218 & 57.65 & $27.78 \mathrm{~dB}$ \\
$16 \times 16$ Array & 20,855 & $-55.39 \mathrm{~dB}$ & 13,010 & 62.38 & $28.35 \mathrm{~dB}$ \\
\hline
\end{tabular}

\section{Conclusions}

In this work, we propose a new distance isolation method to enable the coexistence of FSS ES and BSs. In the proposed distance protection method, the size of the exclusion zone is different for various scenarios ( $\mathrm{RMa}>\mathrm{UMa}>\mathrm{UMi}$ ), where higher elevation angles imply larger off-boresight angles. This means that lower ES antenna gains toward the north side cause lower interferences on the north side in addition to a smaller exclusion zone. Compared to using an omnidirectional antenna, the use of an antenna array allows a larger exclusion zone at the north side of the ES. This is because the maximum gain of the antenna array is higher than that of the omnidirectional antenna. However, on the south side of the ES, the exclusion zone is much smaller because of the directional attenuation pattern of the antenna array. The exclusion zone can be enlarged by increasing the number of antenna elements. The UMi has the largest restriction zone, smallest exclusion zone, and the highest required mean Tx power reduction because of its small ISD value. In the UMa and UMi, the mean Tx power reductions are lower in cases where antenna arrays are used. This is because using the antenna array allows many of the cells to only reduce their Tx power by $1 \mathrm{~dB}$. However, in the case of using an omnidirectional antenna, reducing the $\mathrm{Tx}$ power by $1 \mathrm{~dB}$ barely makes any difference, and most of the BSs need to further decrease their Tx power. Hence, the proposed power control scheme is more efficient in cases where the antenna array is used in the UMa and UMi. Additionally, the proposed scheme and conclusions may help the actual 5G network design and deployment when the issue of 
coexistence between 5G system and satellite system should be solved. Firstly, the exclusion zone and restriction zone can be achieved by loading the actual parameters of ES and 5G BSs. In this way, it is clear where no BSs can exist and where BSs may can be deployed. Next, with the proposed power control scheme, the maximum Tx power of each BSs in the restriction zone can be achieved either by using the 3GPP network layout or customized BSs site selection.

For the future, we will focus on developing angular protection measures to mitigate the interfering beams, decrease the sizes of the exclusion and restriction zones, and decrease the BS Tx power reduction within the restriction zone. Additionally, hybrid technologies can be considered for the future generation system, such as $6 \mathrm{G}$, since non-terrestrial network will become a part of it.

Author Contributions: Y.W. and S.-H.H. contributed to the main idea of this research work. Y.W. wrote computation codes, performed the simulations, experiments, and database collection. Y.W. and S.-H.H. confirmed numerical results of the work. The research activity was planned and executed under the supervision of S.-H.H., Y.W., S.L. and S.-H.H. contributed to the writing of this article. All authors have read and agreed to the published version of the manuscript.

Funding: This work was supported by Electronics and Telecommunications Research Institute (ETRI) grant funded by the Korean government (6011-2018-00016, A study on the supply of 5G additional frequency).

Conflicts of Interest: The authors declare no conflict of interest regarding the publication of this article.

\section{References}

1. Anisimoff. 5G Bands in South Korea. Available online: http://anisimoff.org/eng/5g/spectrum/5g_south_korea.html (accessed on 13 May 2021).

2. Hattab, G.; Moorut, P.; Visotsky, E.; Cudak, M.; Ghosh, A. Interference Analysis of the Coexistence of 5G Cellular Networks with Satellite Earth Stations in 3.7-4.2 GHz. In Proceedings of the 2018 IEEE International Conference on Communications Workshops (ICC Workshops), Kansas City, MO, USA, 20-24 May 2018; Institute of Electrical and Electronics Engineers (IEEE): Piscataway, NJ, USA, 2018; pp. 1-6.

3. Guidolin, F.; Nekovee, M.; Badia, L.; Zorzi, M. A Study on the Coexistence of Fixed Satellite Service and Cellular Networks in a mmWave Scenario; Institute of Electrical and Electronics Engineers (IEEE): Piscataway, NJ, USA, 2015; pp. 2444-2449.

4. ITU Radio Regulations, Section IV. Radio Stations and Systems. Available online: https://www.itu.int/dms_pub/itu-r/md/15 /wrs18/sp/R15-WRS18-SP-0003!!PDF-E.pdf (accessed on 13 May 2021).

5. ETRI Metis Project. Intermediate Description of the Spectrum Needs and Usage Principles. D5.1 Deliverable, August 2013. Available online: https:/ / metis2020.com/wp-content/uploads/deliverables/METIS_D5.1_v1.pdf (accessed on 13 May 2021).

6. Sun, Q.; Nan, S. Coexistence Studies between LTE-Hotspot Indoor and Earth Station of Fixed Satellite Service in the Band 34003600MHz. In Proceedings of the 2012 IEEE 11th International Conference on Signal Processing, Beijing, China, 21-25 October 2012; Institute of Electrical and Electronics Engineers (IEEE): Piscataway, NJ, USA, 2012; Volume 3, pp. $2275-2278$.

7. Su, C.; Han, X.; Yan, X.; Zhang, Q.; Feng, Z. Coexistence Analysis between IMT-Advanced System and Fixed Satellite Service System; Institute of Electrical and Electronics Engineers (IEEE): Piscataway, NJ, USA, 2014; pp. 1692-1697.

8. Kim, S.; Visotsky, E.; Moorut, P.; Bechta, K.; Ghosh, A.; Dietrich, C. Coexistence of 5G With the Incumbents in the 28 and 70 GHz Bands. IEEE J. Sel. Areas Commun. 2017, 35, 1254-1268. [CrossRef]

9. Guidolin, F.; Nekovee, M. Investigating Spectrum Sharing between 5G Millimeter Wave Networks and Fixed Satellite Systems. In Proceedings of the 2015 IEEE Globecom Workshops (GC Wkshps), San Diego, CA, USA, 6-10 December 2015; IEEE: Piscataway, NJ, USA, 2015; pp. 1-7.

10. CEPT ECC Report 254. Operational Guidelines for Spectrum Sharing to Support the Implementation of the Current ECC Framework in the 3600-3800 MHz Range. Available online: https:/ / docdb.cept.org/document/958 (accessed on 13 May 2021).

11. ITU-R Recommendation S.465-6. Reference Radiation Pattern of Earth Station Antennas in the Fixed-Satellite Service for Use in Coordination and Interference Assessment in the Frequency Range from 2 to $31 \mathrm{GHz}$. 2010. Available online: https: //iopscience.iop.org/article/10.1088/1742-6596/1169/1/012054/pdf (accessed on 13 May 2021).

12. Karimi, H.R.; Casagni, A.; Gulyaev, A. Spectrum Sharing between the Mobile Service and Existing Fixed and Fixed Satellite Services in the 3.6-3.8 GHz Band. In Proceedings of the 2015 IEEE International Symposium on Dynamic Spectrum Access Networks (DySPAN), Stockholm, Sweden, 29 September-2 October 2018; Institute of Electrical and Electronics Engineers (IEEE): Piscataway, NJ, USA, 2015; pp. 142-153.

13. ITU-R Report S.2368-0. Sharing Studies between International Mobile Telecommunication-Advanced Systems and Geostationary Satellite Networks in the Fixed-Satellite Service in the $3400-4200 \mathrm{MHz}$ and $4500-4800 \mathrm{MHz}$ Frequency Bands in the WRC Study Cycle Leading to WRC-15. 2015. Available online: https:/ / www.itu.int/pub/R-REP-S.2368 (accessed on 13 May 2021). 
14. 3GPP. Study on Channel Model for Frequencies from 0.5 to $100 \mathrm{GHz}$ (Release 15). 3GPP TR 38.901 V15.0.0. 2018. Available online: https:/ / www.etsi.org/deliver/etsi_tr/138900_138999/138901/14.00.00_60/tr_138901v140000p.pdf (accessed on 13 May 2021).

15. ITU-R P.452-15. Prediction Procedure for the Evaluation of Interference between Stations on the Surface of the Earth at Frequencies above about 0.1 GHz. 2013. Available online: https://www.itu.int/rec/R-REC-P.452/en (accessed on 13 May 2021).

16. 3GPP. Radio Frequency (RF) System Scenarios (Release 15). 3GPP TR 36.942 V15.0.0. 2018. Available online: https:/ / portal.3gpp. org/desktopmodules/Specifications/SpecificationDetails.aspx?specificationId=2592 (accessed on 13 May 2021).

17. 3GPP. Evolved Universal Terrestrial Radio Access (E-UTRA): Base Station (BS) Radio Transmission and Reception (Release 15). 3GPP TS 36.104 V15.0.0. 2018. Available online: https://portal.3gpp.org/desktopmodules/Specifications/SpecificationDetails. aspx?specificationId=2412 (accessed on 13 May 2021).

18. 3GPP. NR: Base Station (BS) Radio Transmission and Reception (Release 15). 3GPP TS 38.104 V15.3.0. 2018. Available online: https:/ / www.lp-ats.com/smart-pcb-stacking?https:/ / www.lp-ats.com/smart-pcb-stacking\%3Futm_source\%3DGoogle\%26 utm_medium\%3Dads\%26utm_campaign\%3D12766553251\%26utm_term\%3D515054125127\&gclid=EAIaIQobChMI-cXkj42e8 QIVA3ZgCh05XQiKEAAYASAAEgKc-PD_BwE (accessed on 13 May 2021).

19. 3GPP. Study of Radio Frequency (RF) and Electromagnetic Compatibility (EMC) Requirements for Active Antenna Array sys-tem (AAAS) Base Station (Release 12). 3GPP TR 37.840 V12.1.0. 2013. Available online: https: / www.mvg-world.com/en/ products/emc/emc-antennas/emc-dual-ridge-horn-antenna-eh022?gclid=EAIaIQobChMI8KaWro2e8QIVhnZgCh1cnQN8 EAAYASAAEgKuCvD_BwE (accessed on 13 May 2021).

20. Tang, P.; Zhang, J.; Molisch, A.F.; Smith, P.J.; Shafi, M.; Tian, L. Estimation of the K-Factor for Temporal Fading from SingleSnapshot Wideband Measurements. IEEE Trans. Veh. Technol. 2019, 68, 49-63. [CrossRef]

21. 3GPP. Study on 3D Channel Model for LTE (Release 12). 3GPP TR 36.873 V12.7.0. 2018. Available online: https: / portal.3gpp. org/desktopmodules/Specifications/SpecificationDetails.aspx?specificationId=2574 (accessed on 13 May 2021).

22. ITU-R M.2135. Technical Characteristics of Autonomous Maritime Radio Devices Operating in the Frequency Band 156162.05 MHz. 2019. Available online: https:/ / www.itu.int/rec/R-REC-M.2135/en (accessed on 13 May 2021). 